\title{
A Convex Variational Model for Restoring Blurred Images with Multiplicative Noise
}

\section{Dong, Yiqiu; Tieyong Zeng}

\section{Published in:}

S I A M Journal on Imaging Sciences

Link to article, DOI:

$10.1137 / 120870621$

Publication date:

2013

\section{Document Version}

Publisher's PDF, also known as Version of record

Link back to DTU Orbit

\section{Citation (APA):}

Dong, Y., \& Tieyong Zeng (2013). A Convex Variational Model for Restoring Blurred Images with Multiplicative Noise. S I A M Journal on Imaging Sciences, 6(3), 1598-1625. https://doi.org/10.1137/120870621

\section{General rights}

Copyright and moral rights for the publications made accessible in the public portal are retained by the authors and/or other copyright owners and it is a condition of accessing publications that users recognise and abide by the legal requirements associated with these rights.

- Users may download and print one copy of any publication from the public portal for the purpose of private study or research.

- You may not further distribute the material or use it for any profit-making activity or commercial gain

- You may freely distribute the URL identifying the publication in the public portal 


\title{
A Convex Variational Model for Restoring Blurred Images with Multiplicative Noise*
}

\author{
Yiqiu Dong ${ }^{\dagger}$ and Tieyong Zeng ${ }^{\ddagger}$
}

\begin{abstract}
In this paper, a new variational model for restoring blurred images with multiplicative noise is proposed. Based on the statistical property of the noise, a quadratic penalty function technique is utilized in order to obtain a strictly convex model under a mild condition, which guarantees the uniqueness of the solution and the stabilization of the algorithm. For solving the new convex variational model, a primal-dual algorithm is proposed, and its convergence is studied. The paper ends with a report on numerical tests for the simultaneous deblurring and denoising of images subject to multiplicative noise. A comparison with other methods is provided as well.
\end{abstract}

Key words. convexity, deblurring, multiplicative noise, primal-dual algorithm, total variation regularization, variational model

AMS subject classifications. 52A41, 65K10, 65K15, 90C30, 90C47

DOI. $10.1137 / 120870621$

1. Introduction. In real applications, degradation effects are unavoidable during image acquisition and transmission. For instance, the photos produced by astronomical telescopes are often blurred by atmospheric turbulence. In order to further improve image processing tasks, image deblurring and denoising continue to attract attention in the applied mathematics community. Based on the imaging systems, various kinds of noise were considered, such as additive Gaussian noise, impulse noise, and Poisson noise. We refer the reader to $[17,21,35$, $40,44,45]$ and references therein for an overview of those noise models and the restoration methods. However, multiplicative noise is a different noise model, and it commonly appears in synthetic aperture radar (SAR), ultrasound imaging, laser images, and so on [8, 9, 39, 43]. For a mathematical description of such degradations, suppose that an image $\hat{u}$ is a real function defined on $\Omega$, a connected bounded open subset of $\mathbb{R}^{2}$ with compact Lipschitz boundary, i.e., $\hat{u}: \Omega \rightarrow \mathbb{R}$. The degraded image $f$ is given by

$$
f=(A \hat{u}) \eta
$$

where $A \in \mathcal{L}\left(L^{2}(\Omega)\right)$ is a known linear and continuous blurring operator and $\eta \in L^{2}(\Omega)$ represents multiplicative noise with mean 1 . Here, $f$ is obtained from $\hat{u}$, which is blurred by the blurring operator $A$, and then is corrupted by the multiplicative noise $\eta$. Usually we assume that $f>0$. In this paper, we concentrate on the assumption that $\eta$ follows a

\footnotetext{
${ }^{*}$ Received by the editors March 20, 2012; accepted for publication (in revised form) May 29, 2013; published electronically August 22, 2013. This work was supported by RGC 211710, RGC 211911, NSFC 11271049, and the FRGs of Hong Kong Baptist University.

http://www.siam.org/journals/siims/6-3/87062.html

${ }^{\dagger}$ Department of Applied Mathematics and Computer Science, Technical University of Denmark, 2800 Kgs. Lyngby, Denmark (yido@dtu.dk).

${ }^{\ddagger}$ Department of Mathematics, Hong Kong Baptist University, Kowloon Tong, Hong Kong (zeng@hkbu.edu.hk).
} 1598

Copyright ( $)$ by SIAM. Unauthorized reproduction of this article is prohibited. 
Gamma distribution, which commonly occurs in SAR. The deblurring process under noise is well known to be an ill-posed problem in the sense of Hadamard [30]. Since the degraded image provides only partial restrictions on the original data, there exist various solutions which can match the observed degraded image under the given blurring operator. Hence, in order to utilize variational methods, the main challenge in image restoration is to design a reasonable and easily solved optimization problem based on the degradation model and the prior information on the original image.

Until the past decade, a few variational methods have been proposed to handle the restoration problem with the multiplicative noise. Given the statistical properties of the multiplicative noise $\eta$, in [39] the recovery of the image $\hat{u}$ was based on solving the following constrained optimization problem:

$$
\begin{array}{ll}
\inf _{u \in S(\Omega)} & \int_{\Omega}|D u| \\
\text { subject to } & \int_{\Omega} \frac{f}{A u} \mathrm{~d} x=1, \\
& \int_{\Omega}\left(\frac{f}{A u}-1\right)^{2} \mathrm{~d} x=\theta^{2},
\end{array}
$$

where $\theta^{2}$ denotes the variance of $\eta, S(\Omega)=\{v \in B V(\Omega): v>0\}, B V(\Omega)$ is the space of functions of bounded variation (see [6] or below), and the total variation (TV) of $u$ is utilized as the objective function in order to preserve significant edges in images. In (1.2), only basic statistical properties, the mean and the variance, of the noise $\eta$ are considered, which somehow limits the restored results. For this reason, based on the Bayes rule and Gamma distribution with mean 1, by using a maximum a posteriori (MAP) estimator, Aubert and Aujol [5] introduced a variational model as follows:

$$
\inf _{u \in S(\Omega)} \int_{\Omega}\left(\log (A u)+\frac{f}{A u}\right) \mathrm{d} x+\lambda \int_{\Omega}|D u|,
$$

where the TV of $u$ is utilized as the regularization term and $\lambda>0$ is the regularization parameter which controls the trade-off between a good fit of $f$ and a smoothness requirement due to the TV regularization. Below we refer to (1.3) as the AA model. Since both (1.2) and (1.3) are nonconvex, the gradient projection algorithms proposed in [39] and [5] may stick at some local minimizers, and the restored results strongly rely on the initialization and the numerical schemes. To overcome this problem and provide a convex model, in [32] Huang, Ng, and Wen introduced an auxiliary variable $z=\log u$ in (1.3), and in [41] Shi and Osher modified (1.3) by adding a quadratic term. With convex models, these two methods both provide better restored results than the method proposed in [5], and they are independent of the initial estimations. In addition, Steidl and Teubner [42] combined the I-divergence as the data fidelity term with the TV regularization or the nonlocal means to remove the multiplicative Gamma noise. A general patch-based denoising filter was proposed in [20]. In [22], the denoising problem was handled by using a $L^{1}$ fidelity term on frame coefficients. In [36], the approach with spatially varying regularization parameters in the AA model (1.3) was considered in order to restore more texture details of the denoised image. In [31], the multiplicative noise

Copyright $\odot$ by SIAM. Unauthorized reproduction of this article is prohibited. 
removal was addressed via a learned dictionary, and extensive experimental results illustrated the leading performance of this approach. However, all of the above methods work only on the multiplicative noise removal issue, and it is still an open question to extend them to the deblurring case.

In this paper, we focus on the restoration of images that are simultaneously blurred and corrupted by multiplicative noise. Since the nonconvexity of the model (1.3) proposed in [5] causes a uniqueness problem and the issue of convergence of the numerical algorithm, we introduce a new convex model by adding a quadratic penalty term based on the statistical properties of the multiplicative Gamma noise. Furthermore, we study the existence and uniqueness of a solution to the new model on the continuous, i.e., functional space, level. Here, we still use the TV regularization in order to preserve edges during the reconstruction. Evidently, it can be readily extended to some other modern regularization terms such as nonlocal TV [26] or the framelet approach [12]. The minimization problem in our method is solved by the primal-dual algorithm proposed in [15, 24] instead of the gradient projection method in $[5,39]$. The numerical results in this paper show that our method has the potential to outperform the other approaches in multiplicative noise removal with deblurring simultaneously.

The rest of the paper is organized as follows. In section 2, we briefly review the TV regularization and provide its main properties. In section 3, based on the statistical properties of the multiplicative Gamma noise, we propose a new convex model for denoising and study the existence and uniqueness of a solution with several other properties. Then in section 4 we extend the model and those properties to the case of denoising and deblurring simultaneously. Section 5 gives the primal-dual algorithm for solving our restoration model based on the work proposed in [15]. The numerical results shown in section 6 demonstrate the efficiency of the new method. Finally, conclusions are drawn in section 7 .

2. Review of TV regularization. In order to preserve significant edges in images, in their seminal work [40] Rudin, Lions, and Osher, introduced TV regularization into image restoration. In this approach, they recover the image in $B V(\Omega)$, which denotes the space of functions of bounded variation (BV); i.e., $u \in B V(\Omega)$ iff $u \in L^{1}(\Omega)$ and the BV-seminorm,

$$
\int_{\Omega}|D u|:=\sup \left\{\int_{\Omega} u \cdot \operatorname{div}(\xi(x)) \mathrm{d} x \mid \xi \in C_{0}^{\infty}\left(\Omega, \mathbb{R}^{2}\right),\|\xi\|_{L^{\infty}\left(\Omega, \mathbb{R}^{2}\right)} \leq 1\right\},
$$

is finite. The space $B V(\Omega)$ endowed with the norm $\|u\|_{B V}=\|u\|_{L^{1}}+\int_{\Omega}|D u|$ is a Banach space. If $u \in B V(\Omega)$, the distributional derivative $D u$ is a bounded Radon measure, and the above quantity defined in (2.1) corresponds to the total variation (TV). Based on the compactness of $B V(\Omega)$, in the two-dimensional case we have the embedding $B V(\Omega) \hookrightarrow L^{p}(\Omega)$ for $1 \leq p \leq 2$, which is compact for $p<2$. See [3, 6, 16] for more details.

3. A convex multiplicative denoising model. To propose a convex multiplicative denoising model, we start from the multiplicative Gamma noise. Suppose that the random variable $\eta$ follows a Gamma distribution; i.e., its probability density function (PDF) is

$$
p_{\eta}(x ; \theta, K)=\frac{1}{\theta^{K} \Gamma(K)} x^{K-1} e^{-\frac{x}{\theta}} \quad \text { for } \quad x \geq 0,
$$

Copyright (c) by SIAM. Unauthorized reproduction of this article is prohibited. 
where $\Gamma$ is the usual Gamma-function, and $\theta$ and $K$ denote the scale and shape parameters, respectively, in the Gamma distribution. Furthermore, the mean of $\eta$ is $K \theta$, and the variance of $\eta$ is $K \theta^{2}$; see [29]. As multiplicative noise, in general we assume that the mean of $\eta$ equals 1; then we have that $K \theta=1$ and its variance is $\frac{1}{K}$.

Now, set a random variable $Y=\frac{1}{\sqrt{\eta}}$, whose PDF is

$$
p_{Y}(y)=\frac{2}{\theta^{K} \Gamma(K)} y^{-2 K-1} e^{-\frac{1}{\theta y^{2}}} \quad \text { for } \quad y \geq 0 .
$$

We can prove the following properties of $Y$.

Proposition 3.1. Suppose that the random variable $\eta$ follows a Gamma distribution with mean 1. Set $Y=\frac{1}{\sqrt{\eta}}$. Then the means of $Y$ and $Y^{2}$ are

$$
\mathbb{E}(Y)=\frac{\sqrt{K} \Gamma(K-1 / 2)}{\Gamma(K)} \quad \text { and } \quad \mathbb{E}\left(Y^{2}\right)=\frac{K}{K-1},
$$

respectively. Furthermore, we have

$$
\mathbb{E}\left(Y^{n}\right)=\frac{K^{\frac{n}{2}} \Gamma\left(K-\frac{n}{2}\right)}{\Gamma(K)}
$$

for any $n \in \mathbb{N}$.

Proof. Based on the PDF of $\eta$ shown in (3.1) and $K \theta=1$, we obtain

$$
\begin{aligned}
\mathbb{E}(Y) & =\int_{0}^{+\infty} \frac{1}{\sqrt{x}} \frac{1}{\theta^{K} \Gamma(K)} x^{K-1} e^{-\frac{x}{\theta}} \mathrm{d} x \\
& =\frac{\Gamma(K-1 / 2)}{\sqrt{\theta} \Gamma(K)} \int_{0}^{+\infty} \frac{1}{\theta^{K-1 / 2} \Gamma(K-1 / 2)} x^{K-3 / 2} e^{-\frac{x}{\theta}} \mathrm{d} x \\
& =\frac{\Gamma(K-1 / 2)}{\sqrt{\theta} \Gamma(K)} \int_{0}^{+\infty} p_{\eta}(x ; \theta, K-1 / 2) \mathrm{d} x \\
& =\frac{\sqrt{K} \Gamma(K-1 / 2)}{\Gamma(K)} .
\end{aligned}
$$

Similarly, we have

$$
\begin{aligned}
\mathbb{E}\left(Y^{2}\right) & =\int_{0}^{+\infty} \frac{1}{x} \frac{1}{\theta^{K} \Gamma(K)} x^{K-1} e^{-\frac{x}{\theta}} \mathrm{d} x \\
& =\frac{\Gamma(K-1)}{\theta \Gamma(K)} \int_{0}^{+\infty} \frac{1}{\theta^{K-1} \Gamma(K-1)} x^{K-2} e^{-\frac{x}{\theta}} \mathrm{d} x \\
& =\frac{K}{K-1}
\end{aligned}
$$

Further, for any $n \in \mathbb{N}$ we can calculate $\mathbb{E}\left(Y^{n}\right)$ similarly, and obtain

$$
\mathbb{E}\left(Y^{n}\right)=\frac{K^{\frac{n}{2}} \Gamma\left(K-\frac{n}{2}\right)}{\Gamma(K)} .
$$

Copyright $($ ) by SIAM. Unauthorized reproduction of this article is prohibited. 
Table 1

The values of $\mathbb{E}\left((Y-1)^{2}\right)$ and the $K L$ divergence of $Y$ with the Gaussian distribution $\mathcal{N}\left(\mu_{K}, \sigma_{K}^{2}\right)$ for different values of $K$.

\begin{tabular}{|c|c|c|c|c|c|}
\hline$K$ & 4 & 6 & 10 & 20 & 30 \\
\hline $\mathbb{E}\left((Y-1)^{2}\right)$ & 0.1178 & 0.0631 & 0.0320 & 0.0141 & 0.0090 \\
\hline$D_{\mathrm{KL}}\left(Y|| \mathcal{N}\left(\mu_{K}, \sigma_{K}^{2}\right)\right)$ & 0.0114 & 0.0068 & 0.0035 & 0.0014 & $8.5739 \mathrm{e}-04$ \\
\hline
\end{tabular}

According to the properties of the Gamma function [4] and Proposition 3.1, if $K$ is large, the mean of $Y$ is close to 1 . Furthermore, we have the following result.

Proposition 3.2. Suppose that the random variable $\eta$ follows a Gamma distribution with mean 1. Set $Y=\frac{1}{\sqrt{\eta}}$. Then

$$
\lim _{K \rightarrow+\infty} E\left((Y-1)^{2}\right)=0 .
$$

Proof. With Proposition 3.1, we readily have

$$
\mathbb{E}\left((Y-1)^{2}\right)=\frac{K}{K-1}-\frac{2 \sqrt{K} \Gamma(K-1 / 2)}{\Gamma(K)}+1 .
$$

Based on the property of the Gamma function (see [4]),

$$
\lim _{K \rightarrow+\infty} \frac{\Gamma(K+\alpha)}{\Gamma(K) K^{\alpha}}=1 \quad \forall \alpha \in \mathbb{R}
$$

taking $\alpha=-\frac{1}{2}$, we can immediately obtain

$$
\lim _{K \rightarrow+\infty} \mathbb{E}\left((Y-1)^{2}\right)=0 .
$$

Proposition 3.2 ensures that the value of $\mathbb{E}\left((Y-1)^{2}\right)$ is small with a large $K$. On the other hand, from Table 1 we can see that, even with a small $K$, its value is still rather small.

Remark 3.3. Since $p_{Y}(y)$ defined in (3.2) is monotonic on either side of the single maximal point $y=1-\frac{\theta}{2}$, it is a strictly unimodal function. In statistics, a Gaussian distribution is commonly used to approximate the unimodal or other distributions; see [23, 38]. To further understand the relationship between $Y$ and a Gaussian distribution, we need the concept of the Kullback-Leibler (KL) divergence. Suppose that $P$ and $Q$ are continuous random variables with the PDFs $p(x)$ and $q(x)$, respectively. The KL divergence of $P$ and $Q$ is defined as

$$
D_{\mathrm{KL}}(P \| Q):=\int_{-\infty}^{\infty} \log \left(\frac{p(x)}{q(x)}\right) p(x) \mathrm{d} x,
$$

which is always nonnegative and is zero iff $P=Q$ almost everywhere; see [7]. Based on the PDF of the random variable $Y$, we can prove that the KL divergence of $Y$ with the Gaussian distribution $\mathcal{N}\left(\mu_{K}, \sigma_{K}^{2}\right)$ tends to 0 when $K$ goes to infinity, where $\mu_{K}$ and $\sigma_{K}^{2}$ are the mean and variance of $Y$, respectively; i.e.,

$$
\mu_{K}:=\mathbb{E}(Y) \quad \text { and } \quad \sigma_{K}:=\sqrt{\mathbb{E}\left(Y^{2}\right)-(\mathbb{E}(Y))^{2}} .
$$

Proposition 3.4. Suppose that the random variable $\eta$ follows a Gamma distribution with mean 1 . Set $Y=\frac{1}{\sqrt{\eta}}$. Then we have the following:

Copyright (C) by SIAM. Unauthorized reproduction of this article is prohibited. 


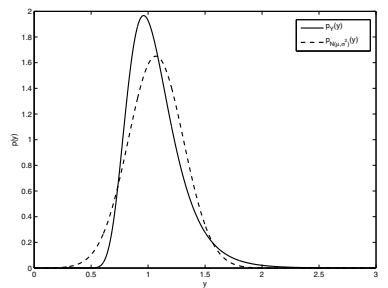

(a)

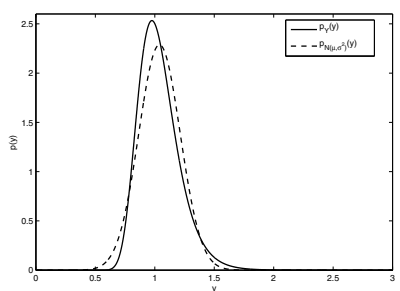

(b)

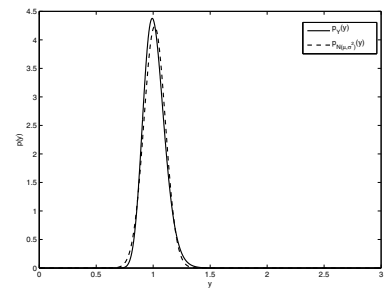

(c)

Figure 1. Comparisons of the PDFs of $Y$ and $\mathcal{N}\left(\mu_{K}, \sigma_{K}^{2}\right)$ with different $K$. (a) $K=6$, (b) $K=10$, (c) $K=30$.

(i) $\int_{0}^{+\infty} p_{Y}(y) \log p_{Y}(y) \mathrm{d} y=\log 2-\log (\sqrt{K} \Gamma(K))+\frac{2 K+1}{2} \psi(K)-K$, where $\psi(K):=$ $\frac{\mathrm{d} \log \Gamma(K)}{\mathrm{d} K}$ is the digamma function (see [2]);

(ii) $\int_{0}^{+\infty} p_{Y}(y) \log p_{\mathcal{N}\left(\mu_{K}, \sigma_{K}^{2}\right)}(y) \mathrm{d} y=-\frac{1}{2} \log \left(2 \pi e \sigma_{K}^{2}\right)$, where $p_{\mathcal{N}\left(\mu_{K}, \sigma_{K}^{2}\right)}(y)$ denotes the PDF of the Gaussian distribution $\mathcal{N}\left(\mu_{K}, \sigma_{K}^{2}\right)$;

(iii) $\lim _{K \rightarrow+\infty} D_{\mathrm{KL}}\left(Y \| \mathcal{N}\left(\mu_{K}, \sigma_{K}^{2}\right)\right)=0$.

Proof. For the proof, see Appendix I (section 8).

Furthermore, let us consider a rescaled version of $Y$, the random variable $Z:=\frac{Y-\mu_{K}}{\sigma_{K}}$. When $K$ goes to infinity, $Y$ degenerates to 1 ; however, $Z$ tends to follow the Gaussian distribution $\mathcal{N}(0,1)$. Further, based on Proposition 3.4, we have

$$
\lim _{K \rightarrow+\infty} D_{\mathrm{KL}}(Z \| \mathcal{N}(0,1))=\lim _{K \rightarrow+\infty} D_{\mathrm{KL}}\left(Y \| \mathcal{N}\left(\mu_{K}, \sigma_{K}^{2}\right)\right)=0 .
$$

Using Proposition 3.4 and the definition of the KL divergence, in Table 1 we list the KL divergence between $Y$ and the Gaussian distribution $\mathcal{N}\left(\mu_{K}, \sigma_{K}^{2}\right)$ with respect to different values of $K$. Obviously, even with small $K, D_{\mathrm{KL}}\left(Y|| \mathcal{N}\left(\mu_{K}, \sigma_{K}^{2}\right)\right)$ is already very small. In addition, in Figure 1 we show the PDFs of $Y$ and $\mathcal{N}\left(\mu_{K}, \sigma_{K}^{2}\right)$ with $K=6, K=10$, and $K=30$. We can see that the PDF of $Y$ decreases quickly and is close to symmetry as $K$ increases, which are the essential properties of Gaussian distribution.

In the denoising case, that is, where $A$ is the identity operator, from the degradation model (1.1) we obtain that $Y=\frac{1}{\sqrt{\eta}}=\sqrt{\frac{u}{f}}$. Inspired by (3.3), we introduce a quadratic penalty term into the AA model (1.3), which turns out to be

$$
\inf _{u \in \bar{S}(\Omega)} E(u):=\int_{\Omega}\left(\log u+\frac{f}{u}\right) \mathrm{d} x+\alpha \int_{\Omega}\left(\sqrt{\frac{u}{f}}-1\right)^{2} \mathrm{~d} x+\lambda \int_{\Omega}|D u|
$$

with the penalty parameter $\alpha>0$. In addition, we set

$$
\bar{S}(\Omega):=\{v \in B V(\Omega): v \geq 0\}
$$

which is closed and convex, and we define $\log 0=-\infty$ and $\frac{1}{0}=+\infty$. Note that as $f>0$, we need not define $\frac{0}{0}$. 
3.1. Existence and uniqueness of a solution. For the existence and uniqueness of a solution to (3.5), we start with discussing the convexity of the model. Since the quadratic penalty term provides extra convexity, we prove that $E(u)$ in $(3.5)$ is convex if the parameter $\alpha$ satisfies certain condition.

Proposition 3.5. If $\alpha \geq \frac{2 \sqrt{6}}{9}$, then the model (3.5) is strictly convex.

Proof. With $t \in \mathbb{R}^{+}$and a fixed $\alpha$, we define a function $g$ as

$$
g(t):=\log t+\frac{1}{t}+\alpha(\sqrt{t}-1)^{2} .
$$

Easily, we have that the second order derivative of $g$ satisfies

$$
g^{\prime \prime}(t)=-t^{-2}+2 t^{-3}+\frac{\alpha}{2} t^{-\frac{3}{2}} .
$$

A direct computation shows that the function $t^{\frac{3}{2}} g^{\prime \prime}(t)$ reaches its unique minimum, $\frac{9 \alpha-2 \sqrt{6}}{18}$, at $t=6$. Hence, if $\alpha \geq \frac{2 \sqrt{6}}{9}$, we have $g^{\prime \prime}(t) \geq 0$; i.e., $g$ is convex. Furthermore, since the function $g$ has only one minimizer, $g$ is strictly convex when $\alpha \geq \frac{2 \sqrt{6}}{9}$.

Setting $t=\frac{u(x)}{f(x)}$ for each $x \in \Omega$, we obtain the strict convexity of the first two terms in (3.5). Based on the convexity of the TV regularization, we deduce that $E(u)$ in (3.5) is strictly convex if $\alpha \geq \frac{2 \sqrt{6}}{9}$. Since the feasible set $\bar{S}(\Omega)$ is convex, the assertion is an immediate consequence.

Based on Proposition 3.5, we see that, with a suitable $\alpha,(3.5)$ is a convex approximation of the nonconvex model (1.3) with $A$ as the identity operator. Now, we argue the existence and uniqueness of a solution to (3.5) and the minimum-maximum principle.

Theorem 3.6. Let $f$ be in $L^{\infty}(\Omega)$ with $\inf _{\Omega} f>0$; then the model (3.5) has a solution $u^{*}$ in $B V(\Omega)$ satisfying

$$
0<\inf _{\Omega} f \leq u^{*} \leq \sup _{\Omega} f .
$$

Moreover, if $\alpha \geq \frac{2 \sqrt{6}}{9}$, the solution of (3.5) is unique.

Proof. Set $c_{1}:=\inf _{\Omega} f, c_{2}:=\sup _{\Omega} f$, and let

$$
E_{0}(u):=\int_{\Omega}\left(\log u+\frac{f}{u}\right) \mathrm{d} x+\alpha \int_{\Omega}\left(\sqrt{\frac{u}{f}}-1\right)^{2} \mathrm{~d} x .
$$

For each fixed $x \in \Omega$, easily we have $\log t+\frac{f(x)}{t} \geq 1+\log f(x)$ with $t \in \mathbb{R}^{+} \cup\{0\}$. Noting that $E(u)$ is defined in (3.5), we thus have

$$
E(u) \geq \int_{\Omega}\left(\log u+\frac{f}{u}\right) \mathrm{d} x \geq \int_{\Omega}(1+\log f) \mathrm{d} x .
$$

In other words, $E(u)$ in (3.5) is bounded from below, and we can choose a minimizing sequence $\left\{u_{n} \in \bar{S}(\Omega): n=1,2, \ldots\right\}$.

Since for each fixed $x \in \Omega$ the real function on $\mathbb{R}^{+} \cup\{0\}$,

$$
g(t):=\log t+\frac{f(x)}{t}+\alpha\left(\sqrt{\frac{t}{f(x)}}-1\right)^{2},
$$

Copyright $\odot$ by SIAM. Unauthorized reproduction of this article is prohibited. 
is decreasing if $t \in[0, f(x))$ and increasing if $t \in(f(x),+\infty)$, one always has $g(\min (t, M)) \leq$ $g(t)$ with $M \geq f(x)$. Hence, we obtain that

$$
E_{0}\left(\inf \left(u, c_{2}\right)\right) \leq E_{0}(u)
$$

Combining this with $\int_{\Omega}\left|D \inf \left(u, c_{2}\right)\right| \leq \int_{\Omega}|D u|$ (see Lemma 1 in section 4.3 of [34]), we have $E\left(\inf \left(u, c_{2}\right)\right) \leq E(u)$. In the same way we are able to get $E\left(\sup \left(u, c_{1}\right)\right) \leq E(u)$. Hence, we can assume that $0<c_{1} \leq u_{n} \leq c_{2}$, which implies that $u_{n}$ is bounded in $L^{1}(\Omega)$.

As $\left\{u_{n}\right\}$ is a minimizing sequence, we know that $E\left(u_{n}\right)$ is bounded. Furthermore, $\int_{\Omega}\left|D u_{n}\right|$ is bounded, and $\left\{u_{n}\right\}$ is bounded in $B V(\Omega)$. Therefore, there exists a subsequence $\left\{u_{n_{k}}\right\}$ which converges strongly in $L^{1}(\Omega)$ to some $u^{*} \in B V(\Omega)$, and $\left\{D u_{n_{k}}\right\}$ converges weakly as a measure to $D u^{*}[6]$. Since $\bar{S}(\Omega)$ is closed and convex, by the lower semicontinuity of the TV and Fatou's lemma, we get that $u^{*}$ is a solution of the model (3.5), and necessarily $0<c_{1} \leq u^{*} \leq c_{2}$.

Moreover, if $\alpha \geq \frac{2 \sqrt{6}}{9}$, uniqueness follows directly from the strict convexity of the function $E$.

As for Theorem 4.1 in [5], here we also need the assumption $\inf _{\Omega} f>0$, which ensures the lower boundedness of $E(u)$ defined in (3.5). In numerical practice, this assumption is always kept by using $\max (f, \epsilon)$ as the observed image with a very small positive value $\epsilon$.

In [5] a comparison principle was given concerning the model (1.3). With the $\alpha$-term in (3.5), it is satisfied with certain conditions on $\alpha$ as well.

Proposition 3.7. Let $f_{1}$ and $f_{2}$ be in $L^{\infty}(\Omega)$ with $a_{1}>0$ and $a_{2}>0$, where $a_{1}=\inf _{\Omega} f_{1}$ and $a_{2}=\inf _{\Omega} f_{2}$. Further, set $b_{1}=\sup _{\Omega} f_{1}$ and $b_{2}=\sup _{\Omega} f_{2}$. Assume $f_{1}<f_{2}$. Suppose $u_{1}^{*}$ (resp., $u_{2}^{*}$ ) is a solution of (3.5) with $f=f_{1}$ (resp., $f=f_{2}$ ). Then when $\alpha<\frac{a_{1} a_{2}}{b_{1} b_{2}-a_{1} a_{2}}$, we have $u_{1}^{*} \leq u_{2}^{*}$.

Proof. Referring to [5], we define $u \wedge v=\inf (u, v)$ and $u \vee v=\sup (u, v)$. Since $u_{i}^{*}$ is a minimizer of $E(u)$ with $f=f_{i}$, which is defined in (3.5), with respect to $i=1,2$, we have

$$
E\left(u_{1}^{*} \wedge u_{2}^{*}\right)+E\left(u_{1}^{*} \vee u_{2}^{*}\right) \geq E\left(u_{1}^{*}\right)+E\left(u_{2}^{*}\right) .
$$

Based on the result $\int_{\Omega}\left|D\left(u_{1}^{*} \wedge u_{2}^{*}\right)\right|+\int_{\Omega}\left|D\left(u_{1}^{*} \vee u_{2}^{*}\right)\right| \leq \int_{\Omega}\left|D u_{1}^{*}\right|+\int_{\Omega}\left|D u_{2}^{*}\right|$ in $[13,27]$, we get

$$
\begin{aligned}
& \int_{\Omega}\left[\log \left(u_{1}^{*} \wedge u_{2}^{*}\right)+\frac{f_{1}}{u_{1}^{*} \wedge u_{2}^{*}}\right.\left.+\alpha\left(\sqrt{\frac{u_{1}^{*} \wedge u_{2}^{*}}{f_{1}}}-1\right)^{2}\right] \mathrm{d} x \\
&+\int_{\Omega}\left[\log \left(u_{1}^{*} \vee u_{2}^{*}\right)+\frac{f_{2}}{u_{1}^{*} \vee u_{2}^{*}}+\alpha\left(\sqrt{\frac{u_{1}^{*} \vee u_{2}^{*}}{f_{2}}}-1\right)^{2}\right] \mathrm{d} x \\
& \geq \int_{\Omega}\left[\log u_{1}^{*}+\frac{f_{1}}{u_{1}^{*}}+\alpha\left(\sqrt{\left.\left.\frac{u_{1}^{*}}{f_{1}}-1\right)^{2}\right]} \mathrm{d} x\right.\right. \\
&+\int_{\Omega}\left[\log u_{2}^{*}+\frac{f_{2}}{u_{2}^{*}}+\alpha\left(\sqrt{\frac{u_{2}^{*}}{f_{2}}}-1\right)^{2}\right] \mathrm{d} x .
\end{aligned}
$$

Copyright (C) by SIAM. Unauthorized reproduction of this article is prohibited. 
Writing $\Omega=\left\{u_{1}^{*}>u_{2}^{*}\right\} \cup\left\{u_{1}^{*} \leq u_{2}^{*}\right\}$, we easily deduce that

$$
\int_{\left\{u_{1}^{*}>u_{2}^{*}\right\}}\left(f_{1}-f_{2}\right)\left(u_{1}^{*}-u_{2}^{*}\right)\left[\frac{1}{u_{1}^{*} u_{2}^{*}}+\frac{\alpha}{f_{1} f_{2}}-\frac{2 \alpha}{\sqrt{f_{1} f_{2}}\left(\sqrt{f_{1}}+\sqrt{f_{2}}\right)\left(\sqrt{u_{1}^{*}}+\sqrt{u_{2}^{*}}\right)}\right] \geq 0 .
$$

Based on Theorem 3.6, we have $0<a_{1} \leq u_{1}^{*} \leq b_{1}$ and $0<a_{2} \leq u_{2}^{*} \leq b_{2}$, which imply $\frac{1}{u_{1}^{*} u_{2}^{*}} \geq \frac{1}{b_{1} b_{2}}$ and

$$
\frac{2}{\sqrt{f_{1} f_{2}}\left(\sqrt{f_{1}}+\sqrt{f_{2}}\right)\left(\sqrt{u_{1}^{*}}+\sqrt{u_{2}^{*}}\right)}-\frac{1}{f_{1} f_{2}} \leq \frac{2}{\sqrt{a_{1} a_{2}}\left(\sqrt{a_{1}}+\sqrt{a_{2}}\right)^{2}}-\frac{1}{b_{1} b_{2}} \leq \frac{1}{a_{1} a_{2}}-\frac{1}{b_{1} b_{2}} .
$$

Hence, we find that

$$
\frac{1}{u_{1}^{*} u_{2}^{*}}+\frac{\alpha}{f_{1} f_{2}}-\frac{2 \alpha}{\sqrt{f_{1} f_{2}}\left(\sqrt{f_{1}}+\sqrt{f_{2}}\right)\left(\sqrt{u_{1}^{*}}+\sqrt{u_{2}^{*}}\right)}>0,
$$

as soon as $\alpha<\frac{a_{1} a_{2}}{b_{1} b_{2}-a_{1} a_{2}}$. Taking account of $f_{1}<f_{2}$, in this case we deduce that $\left\{u_{1}^{*}>u_{2}^{*}\right\}$ has a zero Lebesgue measure; i.e., $u_{1}^{*} \leq u_{2}^{*}$ a.e. in $\Omega$.

4. The extension to simultaneous deblurring and denoising. The model (3.5) is based on the statistical properties of Gamma distribution and is specifically devoted to multiplicative Gamma noise removal. In this section, we extend it to the simultaneous deblurring and denoising case, i.e., to restoring the image $\hat{u}$ in (1.1) with the blurring operator $A$. The restoration is processed by solving the optimization problem

$$
\inf _{u \in \bar{S}(\Omega)} E_{A}(u):=\int_{\Omega}\left(\log A u+\frac{f}{A u}\right) \mathrm{d} x+\alpha \int_{\Omega}\left(\sqrt{\frac{A u}{f}}-1\right)^{2} \mathrm{~d} x+\lambda \int_{\Omega}|D u|,
$$

where $A \in \mathcal{L}\left(L^{2}(\Omega)\right)$. As a blurring operator, we assume that $A$ is nonnegative; i.e., $A \geq 0$ in short. Then we have $A u \geq 0$ with $u \in \bar{S}(\Omega)$. As in Proposition 3.2, since $A$ is linear, we can readily establish the following convexity result.

Proposition 4.1. If $\alpha \geq \frac{2 \sqrt{6}}{9}$, then the model (4.1) is convex.

4.1. Existence of a solution. Based on the properties of TV and the space of BV functions, we prove the existence and uniqueness of a solution to (4.1).

Theorem 4.2. Recall that $\Omega \subset \mathbb{R}^{2}$ is a connected bounded set with compact Lipschitz boundary. Suppose that $A \in \mathcal{L}\left(L^{2}(\Omega)\right)$ is nonnegative and does not annihilate constant functions; i.e., $A 1 \neq 0$. Let $f$ be in $L^{\infty}(\Omega)$ with $\inf _{\Omega} f>0$; then the model (4.1) admits a solution $u^{*}$. Moreover, if $\alpha \geq \frac{2 \sqrt{6}}{9}$ and $A$ is injective, then the solution is unique.

Proof. Similar to the proof of Theorem 3.6, $E_{A}$ is readily bounded from below. We can choose a minimizing sequence $\left\{u_{n}\right\} \subset \bar{S}(\Omega)$ for (4.1). So $\left\{\int_{\Omega}\left|D u_{n}\right|\right\}$ with $n=1,2, \ldots$ is bounded. Using the Poincaré inequality (see Remark 3.50 of [3]), we obtain

$$
\left\|u_{n}-m_{\Omega}\left(u_{n}\right)\right\|_{2} \leq C \int_{\Omega}\left|D\left(u_{n}-m_{\Omega}\left(u_{n}\right)\right)\right|=C \int_{\Omega}\left|D u_{n}\right|,
$$

Copyright (C) by SIAM. Unauthorized reproduction of this article is prohibited. 
where $m_{\Omega}\left(u_{n}\right)=\frac{1}{|\Omega|} \int_{\Omega} u_{n} \mathrm{~d} x$ and $|\Omega|$ denotes the measure of $\Omega$. Further, $C$ is a constant. Recalling that $\Omega$ is bounded, it follows that $\left\|u_{n}-m_{\Omega}\left(u_{n}\right)\right\|_{2}$ is bounded for each $n$. Since $A \in \mathcal{L}\left(L^{2}(\Omega)\right)$ is continuous, $\left\{A\left(u_{n}-m_{\Omega}\left(u_{n}\right)\right)\right\}$ must be bounded in $L^{2}(\Omega)$ and in $L^{1}(\Omega)$.

On the other hand, according to the boundedness of $E_{A}\left(u_{n}\right)$, for each $n,\left(\sqrt{\frac{A u_{n}}{f}}-1\right)^{2}$ is bounded in $L^{1}(\Omega)$, which implies that $\left\|\frac{A u_{n}}{f}\right\|_{1}$ is bounded; then we obtain that $\left\|A u_{n}\right\|_{1}$ is bounded. Moreover, we have

$$
\left|m_{\Omega}\left(u_{n}\right)\right| \cdot\|A 1\|_{1}=\left\|A\left(u_{n}-m_{\Omega}\left(u_{n}\right)\right)-A u_{n}\right\|_{1} \leq\left\|A\left(u_{n}-m_{\Omega}\left(u_{n}\right)\right)\right\|_{1}+\left\|A u_{n}\right\|_{1} .
$$

Hence, $\left|m_{\Omega}\left(u_{n}\right)\right| \cdot\|A 1\|_{1}$ is bounded. Thanks to $A 1 \neq 0$, we obtain that $m_{\Omega}\left(u_{n}\right)$ is uniformly bounded. Together with the boundedness of $\left\{u_{n}-m_{\Omega}\left(u_{n}\right)\right\}$, this leads to the boundedness of $\left\{u_{n}\right\}$ in $L^{2}(\Omega)$ and thus in $L^{1}(\Omega)$. Since $\bar{S}(\Omega)$ is closed and convex, $\left\{u_{n}\right\}$ is bounded in $\bar{S}(\Omega)$ as well.

Therefore, there exists a subsequence $\left\{u_{n_{k}}\right\}$ which converges weakly in $L^{2}(\Omega)$ to some $u^{*} \in L^{2}(\Omega)$, and $\left\{D u_{n_{k}}\right\}$ converges weakly as a measure to $D u^{*}$. Due to the continuity of the linear operator $A$, one must have that $\left\{A u_{n_{k}}\right\}$ converges weakly to $A u^{*}$ in $L^{2}(\Omega)$. Then, based on the lower semicontinuity of the TV and Fatou's lemma, we obtain that $u^{*}$ is a solution of the model (4.1).

Based on Proposition 4.1, when $\alpha \geq \frac{2 \sqrt{6}}{9}$, the model (4.1) is convex. Furthermore, if $A$ is injective, (4.1) is strictly convex, and then its minimizer has to be unique.

As in Theorem 3.6, the assumption that $\inf _{\Omega} f>0$ basically guarantees the well-posedness of the model. Moreover, in the discrete settings, adding a few more technicalities, the assumption that $A$ is injective may be weakened to $\operatorname{ker}(A) \bigcap \operatorname{ker}(\nabla)=\{0\}$, where $\nabla$ denotes the discrete gradient operator.

Remark 4.3. In the proof of Theorem 4.2, because of the $\alpha$-term, we obtain that the sequence $\left\{A u_{n}\right\}$ is bounded in $L^{1}(\Omega)$. Thus, when $\alpha=0$, that is, in the case of the model (1.3), it is difficult to get the same result, and in [5] the existence of a solution to (1.3) is still an open question.

According to the constraint in (4.1), we find that the model's minimizer is nonnegative. Further, we have the following result.

Proposition 4.4. Suppose that $u^{*}$ is the solution of (4.1). Then there exists a constant $C_{1}$ such that

$$
\left|\left\{x \in \Omega:\left(A u^{*}\right)(x) \leq \epsilon f(x)\right\}\right| \leq \frac{\epsilon}{1+\epsilon \log \epsilon}\left(C_{1}+\int_{\Omega} \log f \mathrm{~d} x\right)
$$

for any $0<\epsilon<1$.

Proof. Suppose that $C_{1}$ is the minimal value of (4.1), which is independent of $\epsilon$. Set $w=\frac{A u^{*}}{f}$; then we have

$$
|\Omega|+\int_{\Omega} \log f \mathrm{~d} x \leq \int_{\Omega}\left(\log w+\frac{1}{w}\right) \mathrm{d} x \leq C_{1}+\int_{\Omega} \log f \mathrm{~d} x,
$$

where we have used the fact that, for each $t>0, \log t+\frac{1}{t} \geq 1$. Moreover, if $t \leq \epsilon<1$, then $\log t+\frac{1}{t} \geq \log \epsilon+\frac{1}{\epsilon}$, and we get that

$$
|\{x \in \Omega: w(x) \leq \epsilon\}| \leq \frac{\epsilon}{1+\epsilon \log \epsilon}\left(C_{1}+\int_{\Omega} \log f \mathrm{~d} x\right) .
$$

Copyright $\odot$ by SIAM. Unauthorized reproduction of this article is prohibited. 
Based on $w=\frac{A u^{*}}{f}$, we obtain the assertion.

As a consequence, $\left|\left\{x \in \Omega:\left(A u^{*}\right)(x)=0\right\}\right|=0$; i.e., $A u^{*}$ is positive a.e. Especially, in the discrete situation, $A u^{*}$ is strictly positive.

4.2. Bias correction. In [22], a variational model was proposed in the log-image domain for multiplicative noise removal. In order to ensure that the mean of the restored image equals that of the observed image, the method ends up an exponential transform along with a bias correction. In addition, we recall that through the theoretical analysis in [14, 16], the classical ROF (Rudin-Osher-Fatemi) model in [40],

$$
\inf _{u \in B V(\Omega)} \int_{\Omega} \frac{1}{2}(A u-f)^{2} \mathrm{~d} x+\lambda \int_{\Omega}|D u|,
$$

is proved to preserve the mean; i.e., $m_{\Omega}\left(A u^{*}\right)=m_{\Omega}(f)$ with $u^{*}$ as a solution. However, (4.3) is proposed to remove the additive white Gaussian noise. In this section, we consider our new model (4.1) through theoretical analysis similar to that in [14] and try to find the relation between the observed image $f$ and the solution $u^{*}$.

Proposition 4.5. Suppose that $A 1=1$. Let $u^{*}$ be a solution of (4.1), and assume that we have $\inf _{\Omega} A u^{*}>0$. Then the following properties hold true:

(i)

$$
\int_{\Omega}\left[\frac{f}{\left(A u^{*}\right)^{2}}-\alpha\left(\frac{1}{f}-\frac{1}{\sqrt{f \cdot A u^{*}}}\right)\right] \mathrm{d} x=\int_{\Omega} \frac{1}{A u^{*}} \mathrm{~d} x .
$$

(ii) If there exists a solution in the case of $\alpha=0$, then we have

$$
\int_{\Omega} \frac{1}{f} \mathrm{~d} x \geq \int_{\Omega} \frac{1}{A u^{*}} \mathrm{~d} x
$$

Proof.

(i) We define a function with single variable $t \in\left(-\inf _{\Omega} A u^{*},+\infty\right)$ :

$$
\begin{aligned}
e(t):= & \int_{\Omega}\left(\log A\left(u^{*}+t\right)+\frac{f}{A\left(u^{*}+t\right)}\right) \mathrm{d} x+\alpha \int_{\Omega}\left(\sqrt{\frac{A\left(u^{*}+t\right)}{f}}-1\right)^{2} \mathrm{~d} x \\
& +\lambda \int_{\Omega}\left|D\left(u^{*}+t\right)\right| .
\end{aligned}
$$

Concerning $A 1=1$, we necessarily have

$$
e(t)=\int_{\Omega}\left(\log \left(A u^{*}+t\right)+\frac{f}{A u^{*}+t}\right) \mathrm{d} x+\alpha \int_{\Omega}\left(\sqrt{\frac{A u^{*}+t}{f}}-1\right)^{2} \mathrm{~d} x+\lambda \int_{\Omega}\left|D u^{*}\right| .
$$

Since $t=0$ is a (local) minimizer of $e(t)$, we have $e^{\prime}(0)=0$, which leads to

$$
\int_{\Omega}\left[\frac{1}{A u^{*}}-\frac{f}{\left(A u^{*}\right)^{2}}+\alpha\left(\frac{1}{f}-\frac{1}{\sqrt{f \cdot A u^{*}}}\right)\right] \mathrm{d} x=0 .
$$

Copyright (C) by SIAM. Unauthorized reproduction of this article is prohibited. 
(ii) With $\alpha=0$, the result in (i) becomes

$$
\int_{\Omega} \frac{f}{\left(A u^{*}\right)^{2}} \mathrm{~d} x=\int_{\Omega} \frac{1}{A u^{*}} \mathrm{~d} x .
$$

Moreover, according to Hölder's inequality and the nonnegativity of $A u^{*}$ and $f$, we obtain

$$
\int_{\Omega} \frac{f}{\left(A u^{*}\right)^{2}} \mathrm{~d} x \cdot \int_{\Omega} \frac{1}{f} \mathrm{~d} x \geq\left(\int_{\Omega} \frac{1}{A u^{*}}\right)^{2} \mathrm{~d} x .
$$

Combining both, we have

$$
\int_{\Omega} \frac{1}{f} \mathrm{~d} x \geq \int_{\Omega} \frac{1}{A u^{*}} \mathrm{~d} x
$$

Note that in the discrete situation the assumption that $\inf _{\Omega} A u^{*}>0$ is always satisfied, based on the conclusion of Proposition 4.4.

From Proposition 4.5 we cannot obtain the similar theoretical result as in $[14,16]$; that is, the mean of the observed image is automatically preserved for the model (4.1). In order to reduce the influence from the bias and keep the restored image in the same scale as $f$, we improve the model (4.1) as

$$
\inf _{\left\{u \in \bar{S}(\Omega): m_{\Omega}(u)=m_{\Omega}(f)\right\}} \int_{\Omega}\left(\log A u+\frac{f}{A u}\right) \mathrm{d} x+\alpha \int_{\Omega}\left(\sqrt{\frac{A u}{f}}-1\right)^{2} \mathrm{~d} x+\lambda \int_{\Omega}|D u| .
$$

It is straightforward to show that the feasible set $\left\{u \in \bar{S}(\Omega): m_{\Omega}(u)=m_{\Omega}(f)\right\}$ is closed and convex, and then the existence and uniqueness of a solution to (4.4) are easily obtained by extending Theorem 4.2. Note that the bias-variance trade-off in statistics does not always advocate for unbiased estimators. It is possible to obtain better peak signal-to-noise ratio (PSNR) results with a small (but different from zero) bias. However, in our numerical simulations, we do observe the improvement of PSNR with our bias correction step.

In (4.4), we implicitly suppose that

$$
m_{\Omega}(u) \approx m_{\Omega}(A u), \quad m_{\Omega}((A u) \eta) \approx m_{\Omega}(A u) .
$$

Under some independence conditions, the above assumptions are theoretically rooted in statistics. Moreover, in the practical simulations, we find that these two assumptions provide rather reasonable results.

5. Primal-dual algorithm. Since the model (4.4) is convex, there are many methods that can be extended to solve the minimization problem in (4.4). For example, the alternating direction method [11, 25], which is convergent and is well suited to large-scale convex problems, and its variant, the split-Bregman algorithm [28], which is widely used to solve $L^{1}$ regularization problems such as the TV regularization. In this section, we apply the primal-dual algorithm proposed in $[15,24,37]$ to solve the minimization problem in (4.4). This algorithm can be used for solving a large family of nonsmooth convex optimization problems; see the applications in [15]. It is simple and comes with the convergence guarantees. 
Now, we focus on the discrete version of (4.4). For the sake of simplicity, we keep the same notation from the continuous context. Then the discrete model reads as follows:

$$
\min _{u \in X} E_{A}(u):=\langle\log A u, 1\rangle+\left\langle\frac{f}{A u}, 1\right\rangle+\alpha\left\|\sqrt{\frac{A u}{f}}-1\right\|_{2}^{2}+\lambda\|\nabla u\|_{1},
$$

where $X=\left\{v \in \mathbb{R}^{n}: v_{i} \geq 0\right.$ for $i=1, \ldots, n$, and $\left.\sum_{i=1}^{n} v_{i}=\sum_{i=1}^{n} f_{i}\right\}, n$ is the number of pixels in the images, $f \in X$ is obtained from a two-dimensional pixel-array by concatenation in the usual columnwise fashion, and $A \in \mathbb{R}^{n \times n}$. Moreover, the vector inner product $\langle u, v\rangle=$ $\sum_{i=1}^{n} u_{i} v_{i}$ is used, and $\|\cdot\|_{2}$ denotes the $l^{2}$-vector-norm. The discrete gradient operator $\nabla \in \mathbb{R}^{2 n \times n}$ is defined by

$$
\nabla v=\left[\begin{array}{c}
\nabla_{x} v \\
\nabla_{y} v
\end{array}\right]
$$

for $v \in \mathbb{R}^{n}$, with $\nabla_{x}, \nabla_{y} \in \mathbb{R}^{n \times n}$ corresponding to the discrete derivatives in the $x$-direction and $y$-direction, respectively. In our numerics, $\nabla_{x}$ and $\nabla_{y}$ are obtained by applying finite difference approximations for the derivatives with symmetric boundary conditions in the respective coordinate directions. In addition, $\|\nabla v\|_{1}$ denotes the discrete TV of $v$, which is defined as

$$
\|\nabla v\|_{1}=\sum_{i=1}^{n} \sqrt{\left(\nabla_{x} v\right)_{i}^{2}+\left(\nabla_{y} v\right)_{i}^{2}}
$$

Define the function $G: X \rightarrow \mathbb{R}$ as

$$
G(u):=\langle\log A u, 1\rangle+\left\langle\frac{f}{A u}, 1\right\rangle+\alpha\left\|\sqrt{\frac{A u}{f}}-1\right\|_{2}^{2} .
$$

Based on the definition of TV in section 2, we give the primal-dual formulation of (5.1):

$$
\max _{p \in Y} \min _{u \in X} G(u)-\lambda\langle u, \operatorname{div} p\rangle
$$

where $Y=\left\{q \in \mathbb{R}^{2 n}:\|q\|_{\infty} \leq 1\right\},\|q\|_{\infty}=\max _{i \in\{1, \ldots, n\}}\left|\sqrt{q_{i}^{2}+q_{i+n}^{2}}\right|$ denotes the $l^{\infty}$-vectornorm, $p$ is the dual variable, and the divergence operator $\operatorname{div}=-\nabla^{\top}$.

This is a generic saddle-point problem, and we can apply the primal-dual algorithm proposed in [15] to solve the above optimization task. The algorithm is summarized as follows.

AlGORITHM FOR SOLVING THE MODEL (5.1).

1: Fixed $\sigma, \tau$. Initialize $u^{0}=f, \bar{u}^{0}=f$, and $p^{0}=(0, \ldots, 0)^{\top} \in \mathbb{R}^{2 n}$.

2: Calculate $p^{k+1}$ and $u^{k+1}$ from

$$
\begin{aligned}
& p^{k+1}=\arg \max _{p \in Y} \lambda\left\langle\bar{u}^{k}, \operatorname{div} p\right\rangle-\frac{1}{2 \sigma}\left\|p-p^{k}\right\|_{2}^{2}, \\
& u^{k+1}=\arg \min _{u \in X} G(u)-\lambda\left\langle u, \operatorname{div} p^{k+1}\right\rangle+\frac{1}{2 \tau}\left\|u-u^{k}\right\|_{2}^{2}, \\
& \bar{u}^{k+1}=2 u^{k+1}-u^{k} .
\end{aligned}
$$

Copyright (C) by SIAM. Unauthorized reproduction of this article is prohibited. 
3: Stop; or set $k:=k+1$ and go to step 2 .

In order to apply the algorithm to (5.1), the main questions are how to solve the dual problem in (5.3) and the primal problem in (5.4). For (5.3), the solution can be easily given by

$$
p_{i}^{k+1}=\pi_{1}\left(\lambda \sigma\left(\nabla \bar{u}^{k}\right)_{i}+p_{i}^{k}\right) \quad \text { for } i=1, \ldots, 2 n,
$$

where $\pi_{1}$ is the projector onto the $l^{2}$-normed unit ball; i.e.,

$$
\pi_{1}\left(q_{i}\right)=\frac{q_{i}}{\max \left(1,\left|q_{i}\right|\right)} \quad \text { and } \quad \pi_{1}\left(q_{n+i}\right)=\frac{q_{n+i}}{\max \left(1,\left|q_{i}\right|\right)} \quad \text { for } i=1, \ldots, n,
$$

with $\left|q_{i}\right|=\sqrt{q_{i}^{2}+q_{i+n}^{2}}$. Since the minimization problem in (5.4) is strictly convex and its objective function has the second derivative, it can be solved efficiently by the Newton method following with one projection step,

$$
u_{i}^{k}:=\frac{\sum_{j=1}^{n} f_{j}}{\sum_{j=1}^{n} \max \left(u_{j}^{k}, 0\right)} \max \left(u_{i}^{k}, 0\right) \quad \text { for } i=1, \ldots, n,
$$

to ensure that $u^{k}$ is nonnegative and preserves the mean of $f$. This projection is inspired by Proposition 2.1 of [18] or Proposition 12 of [19].

Based on Theorem 1 in [15], we end this section with the convergence properties of our algorithm. The proof is given in [15].

Proposition 5.1. The iterates $\left(u^{k}, p^{k}\right)$ of our algorithm converge to a saddle point of (5.2), provided that $\sigma \tau \lambda^{2}\|\nabla\|^{2}<1$.

According to the result $\|\nabla\|^{2} \leq 8$ with the unit spacing size between pixels in [13], we need only $\sigma \tau \lambda^{2}<\frac{1}{8}$ in order to keep the convergent condition. In our numerical practice, $\lambda$ is tuned empirically (and usually it is around 0.1; see next section for details), and we simply set $\sigma=3$ and $\tau=3$, which in most cases ensures the convergence of the algorithm.

6. Numerical results. In this section we provide numerical results to study the behavior of our method with respect to its image restoration capabilities and CPU-time consumption. Here, we compare our method with the one proposed in [5] (AA method) by solving (1.3) and with the one in [39] (RLO method, for the author's initials) by solving (1.2), and both of them are able to remove the multiplicative noise and deblurring simultaneously. In the denoising case, we also provide numerical results from [20], where a probabilistic patch-based (PPB) method was proposed for several kinds of noise, including multiplicative noise. Since in the AA and RLO methods the preservation of the mean of the observed image is not guaranteed, in order to compare fairly, we add the same projection step as in (5.7) before outputting the results. Indeed, assume that $u^{o}$ is the result of the AA method or the RLO method; then we revise it as

$$
u_{i}^{\text {new }}:=\frac{\sum_{j=1}^{n} f_{j}}{\sum_{j=1}^{n} \max \left(u_{j}^{o}, 0\right)} \max \left(u_{i}^{o}, 0\right) \quad \text { for } i=1, \ldots, n .
$$

Note that usually this will improve the result by around 0.1 to $0.3 \mathrm{~dB}$, depending on images and noise levels. For the PPB filter, we numerically find that this correction is not necessary.

Copyright (C) by SIAM. Unauthorized reproduction of this article is prohibited. 


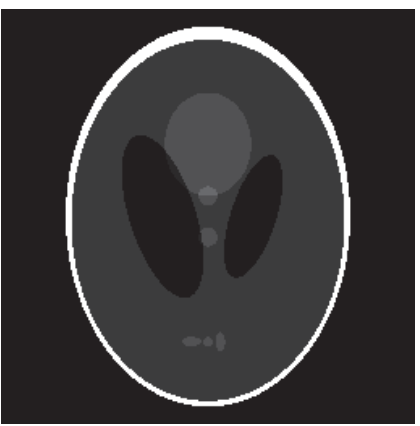

(a)

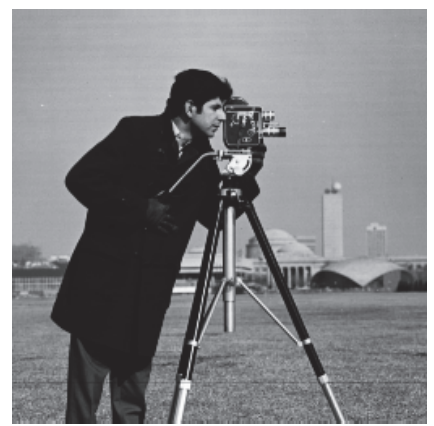

(b)

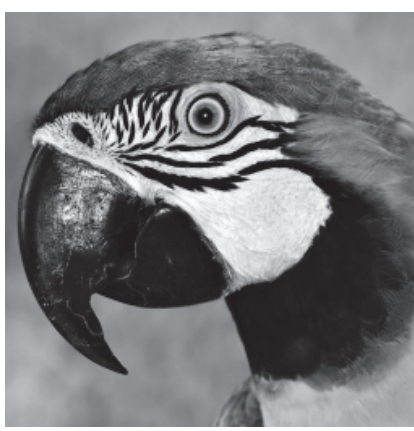

(c)

Figure 2. Original images. (a) "Phantom," (b) "Cameraman," (c) "Parrot."

For illustrations, the results for the 256-by-256 gray level images Phantom, Cameraman, and Parrot are presented; see the original test images in Figure 2. The quality of the restoration results is compared quantitatively by means of the PSNR [10], which is a widely used image quality assessment measure. In addition, all simulations listed here are run in MATLAB 7.12 (R2011a) on a PC equipped with 2.40GHz CPU and 4G RAM memory.

6.1. Image denoising. Although our method is proposed for the simultaneous deblurring and denoising of images subject to multiplicative noise, here we show that it also provides good results for noise removal only. However, since our model is rather basic, more advanced approaches such as the patch-based method [20] or dictionary learning method [31] for multiplicative noise removal could have better results. In our example, the test images are corrupted by multiplicative noise with $K=10$ and $K=6$, respectively. The results are shown in Figures 3-8. For the AA method and the RLO method, we use the time-marching algorithm to solve the minimization models, as proposed in [5,39]. We set the step size to 0.1 in order to obtain a stable iterative procedure. The algorithms are stopped when the maximum number of iterations is reached. In addition, after many experiments with different $\lambda$-values in the AA model, the RLO model, and ours, the ones that provide the best PSNRs are presented here. In the PPB filter [20], we use the codes provided by the authors in [1], which are written as $\mathrm{C}++$ mex-functions. Since the codes were written for removing multiplicative noise with Nakagami-Rayleigh distribution, a square root transform was applied in order to eliminate the gap between the Gamma and Nakagami-Rayleigh distributions. We use the suggested parameter values for the PBB filter except for Phantom, where the search window size has to be enlarged to get better result, which costs more time (see Table 2). In our method, we stop the iterative procedure as soon as the value of the objective function has no big relative decrease, i.e.,

$$
\frac{E\left(u^{k}\right)-E\left(u^{k+1}\right)}{E\left(u^{k}\right)}<\varepsilon .
$$

In the denoising case, we set $\varepsilon=5 \times 10^{-4}$. Notice that the computing time of the PPB filter in [20] is not comparable with the remaining three TV-based methods, since the programming languages are different. 


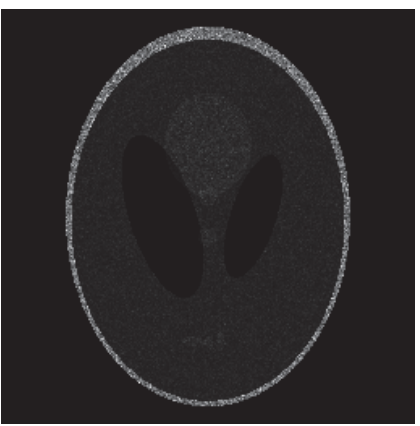

(a)
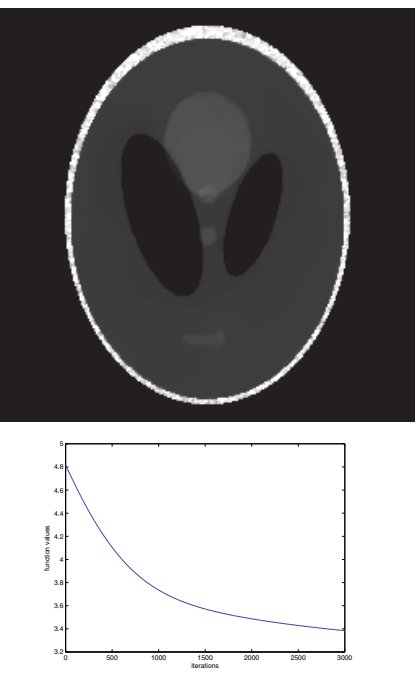

(c)
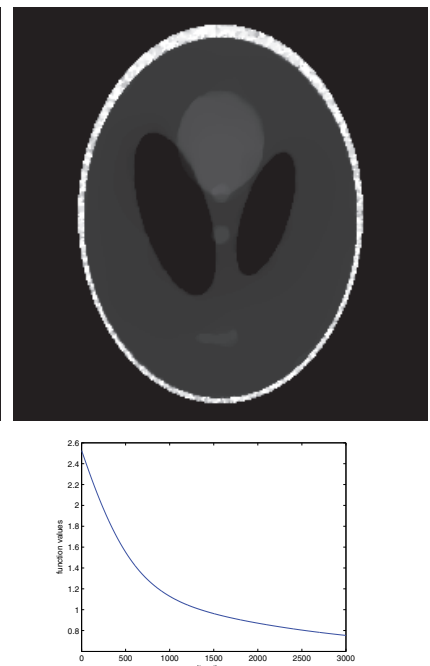

(d)

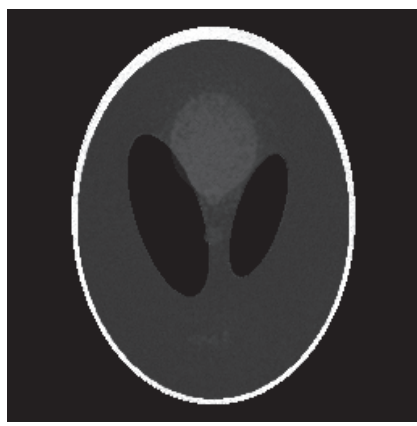

(b)
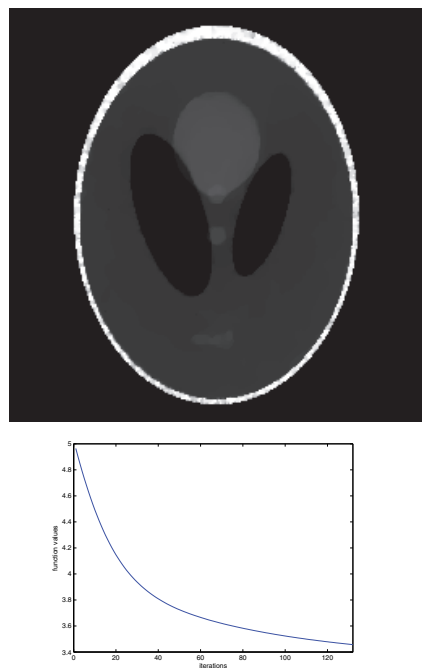

(e)

Figure 3. Results of different methods when removing the multiplicative noise with $K=10$. Bottom row: Plots of the objective function values versus iterations. (a) Noisy Phantom, (b) PPB filter, (c) AA method $(\lambda=0.1)$, (d) RLO method ( $\lambda=0.12)$, (e) our method $(\lambda=0.11$ and $\alpha=8)$.

Comparing the results of the three TV-based methods, i.e., the AA, RLO, and our method in Figures 3-6, we see that all of their objective function values are monotonically decreasing, and our method performs best visually with the fewest iterations. Note that in the restored results for the AA and RLO methods, much more noise remains compared with those of our method; see, e.g., the white boundary of Phantom and the background in Cameraman. Moreover, the contrasts of the details for the AA method and the RLO method are noticeably reduced because of oversmoothing during noise removal; however, our method preserves more details. In this respect, observe the tripod and the trousers in Cameraman, especially when recovering the images corrupted by high-level noise. In order to compare the capability of recovering details, in Figures 7 and 8 we show the results for denoising the image Parrot, which includes more details. Comparing the textures surrounding the eye and the background of the parrot, we can clearly see that our method suppresses noise successfully while preserving significantly more details. 
Table 2

The comparisons of PSNR values, the number of iterations, and CPU-time in seconds for different methods for the denoising case.

\begin{tabular}{|c||c||c|c|c||c|c|c|}
\hline \multirow{2}{*}{ Images } & \multirow{2}{*}{\multicolumn{1}{|c||}{ Methods }} & \multicolumn{3}{c||}{$K=10$} & \multicolumn{3}{c|}{$K=6$} \\
\cline { 3 - 7 } Phantom & PSNR(dB) & Iter & Time(s) & PSNR(dB) & $\sharp$ Iter & Time(s) \\
\hline & AA & 29.44 & 3000 & 30.39 & 27.20 & 3000 & 30.73 \\
& RLO & 29.37 & 3000 & 36.92 & 27.08 & 3000 & 37.77 \\
& PPB & 33.32 & 4 & 43.20 & 29.58 & 4 & 42.17 \\
& Ours & 30.44 & 132 & 6.09 & 28.05 & 174 & 8.24 \\
\hline \multirow{3}{*}{ Cameraman } & AA & 24.38 & 3000 & 31.09 & 23.20 & 3000 & 33.64 \\
& RLO & 24.31 & 3000 & 37.99 & 23.11 & 3000 & 37.88 \\
& PPB & 26.26 & 4 & 16.43 & 25.22 & 4 & 15.11 \\
& Ours & 25.01 & 162 & 8.50 & 23.85 & 168 & 11.82 \\
\hline \multirow{3}{*}{ Parrot } & AA & 24.53 & 3000 & 27.86 & 23.23 & 3000 & 29.14 \\
& RLO & 24.28 & 3000 & 36.60 & 22.96 & 3000 & 39.61 \\
& PPB & 25.52 & 4 & 15.64 & 24.73 & 4 & 14.41 \\
& Ours & 25.47 & 179 & 9.19 & 24.21 & 219 & 11.70 \\
\hline
\end{tabular}

Since the PPB filter in [20] is a patch-based method, which has a framework different from that of the TV-based methods, it can remove the multiplicative noise more effectively and preserve more details. But we still find that some unpleasant artifacts are noticeable in the results obtained by the PPB filter; see, e.g., the homogeneous regions in Cameraman, Phantom, and Parrot. Furthermore, some tiny structures in the images are completely missing; see, e.g., the spots in Phantom and the buildings in Cameraman. Since we utilize TV regularization in our method, those spurious artifacts are absent from our results, but at the same time there might be some undesirable staircasing effects; see the beak and neck of the parrot.

For a comparison of the performance quantitatively and the computational efficiency, in Table 2 we list the PSNR values of the restored results, the number of iterations, and the CPU-times. In the three TV-based methods, we observe that the PSNR values from our method are more than $0.65 \mathrm{~dB}$ higher than others. Due to a large step size, with many fewer iterations our method reaches the stopping rule and also spends much less CPU-time. However, in order to obtain a stable iterative procedure, the AA and RLO methods have to use a small step size, and then need more than 10 times more iterations to provide results with the best PSNRs.

6.2. Image deblurring and denoising. In this section, we consider the restoration of the noisy blurred images. In our experiments, we test two blurring operators, which are motion blur with length 5 and angle 30, and Gaussian blur with a window size $7 \times 7$ and a standard deviation of 2. Further, after blurring, the test images are corrupted by multiplicative noise with $K=10$. In the blurring case, we set $\varepsilon=10^{-4}$ in (6.2) as the stopping rule for our method.

In Figures 9-11, we show the degraded images and the restored results for all three TVbased methods, and Table 3 lists the PSNR values, the number of iterations, and the CPUtimes. In contrast to the results for the AA and RLO methods, our method also performs best both visually and quantitatively, and it preserves more details; see, e.g., the tripod in Cameraman and the texture near the eye in Parrot. Due to the blurring, more iterations are needed in all three methods, but our method still provides the best results in many fewer 


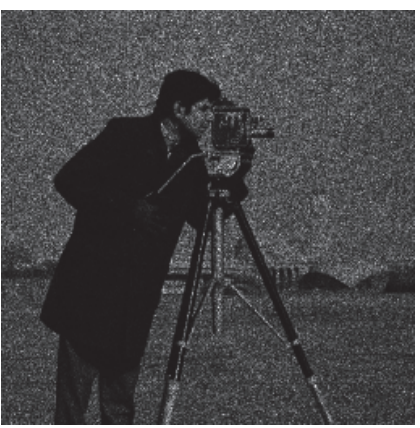

(a)
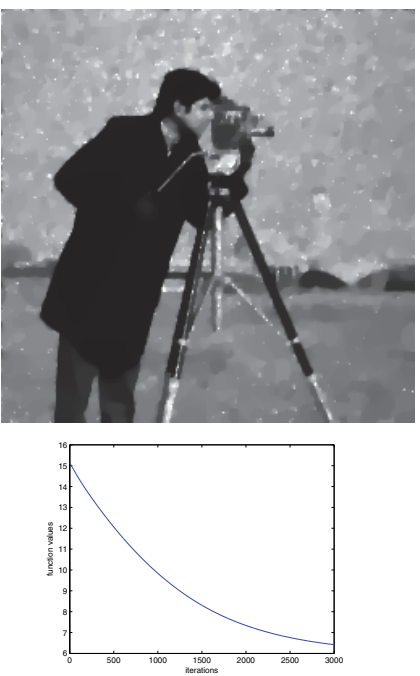

(c)
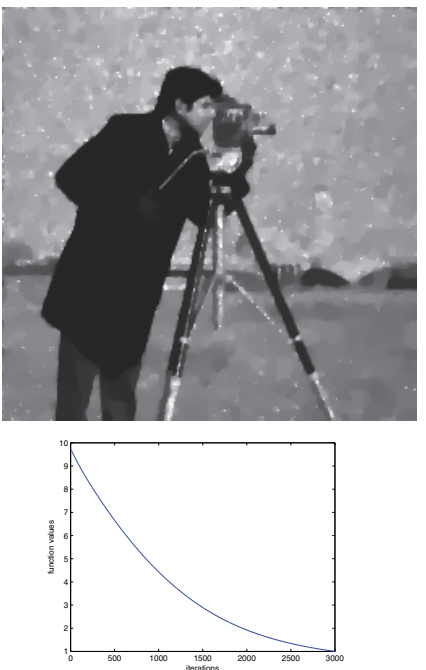

(d)

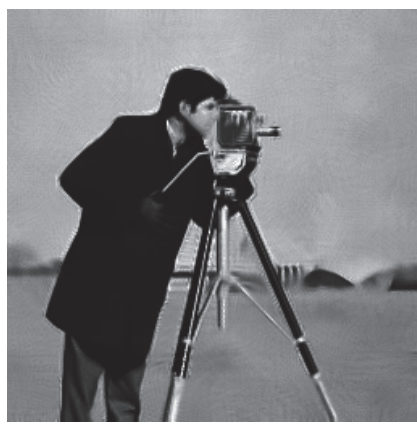

(b)
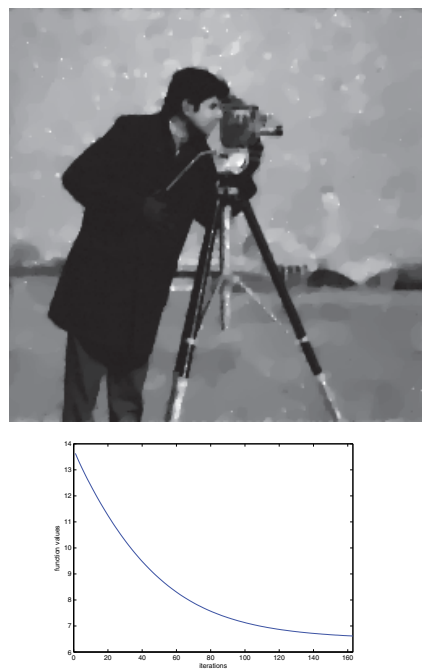

(e)

Figure 4. Results of different methods when removing the multiplicative noise with $K=10$. Bottom row: Plots of the objective function values versus iterations. (a) Noisy Cameraman, (b) PPB filter, (c) AA method $(\lambda=0.14)$, (d) RLO method $(\lambda=0.14)$, (e) our method $(\lambda=0.12$ and $\alpha=16)$.

iterations with the least CPU-times. In conclusion, our method turns out to be more efficient and outperforms the other methods which are able to deblur while removing multiplicative noise simultaneously.

7. Conclusion. In this paper, we propose a new variational model for restoring blurred images subject to multiplicative Gamma noise. In order to obtain the convexity, we add a quadratic penalty term according to the statistical properties of the multiplicative Gamma noise in the model proposed in [5], which combines an MAP estimator with the TV regularization. The existence and uniqueness of a solution to the new model are obtained. Furthermore, some other properties are studied, such as the minimum-maximum principle, bias correction, and so on. Due to the convexity, we are allowed to employ the primal-dual algorithm proposed in [15] to solve the corresponding minimization problem in the new model, and its convergence is guaranteed. Compared to other recently proposed methods, our method appears to be very competitive with respect to image restoration capabilities and CPU-time consumption. 


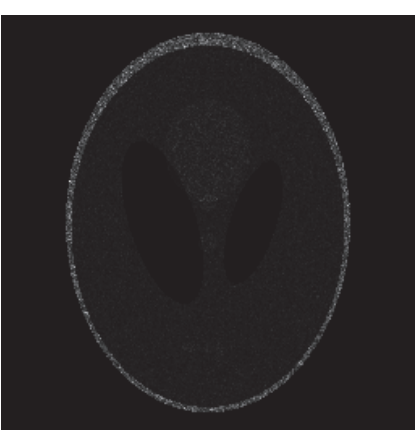

(a)
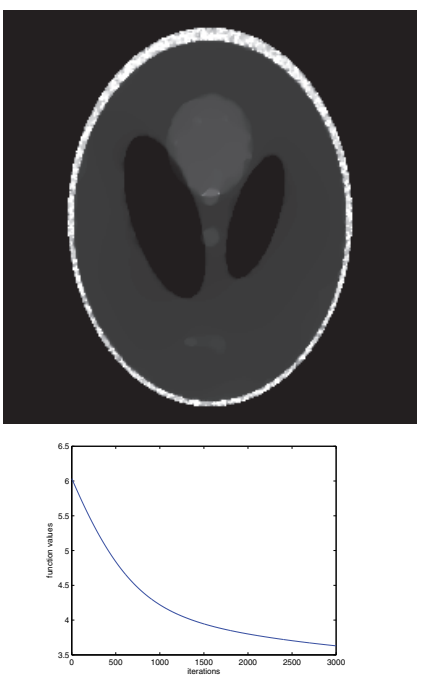

(c)
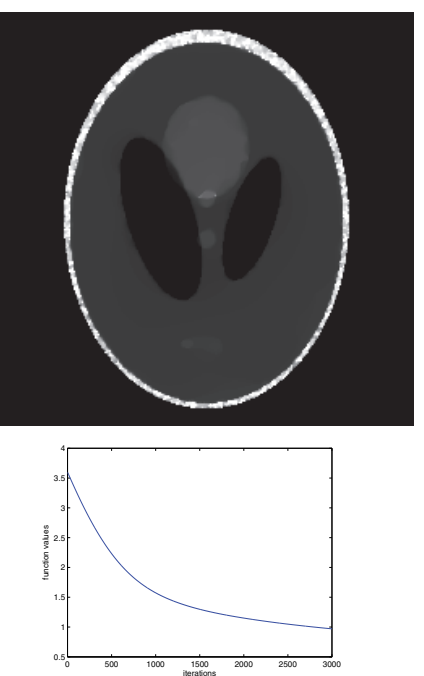

(d)

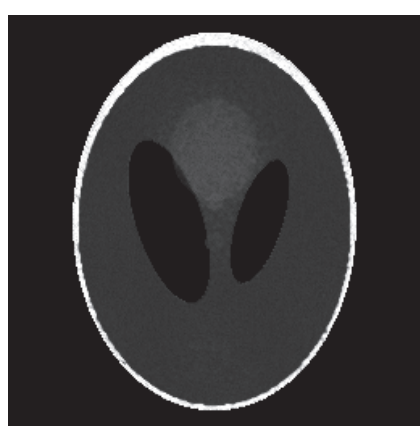

(b)
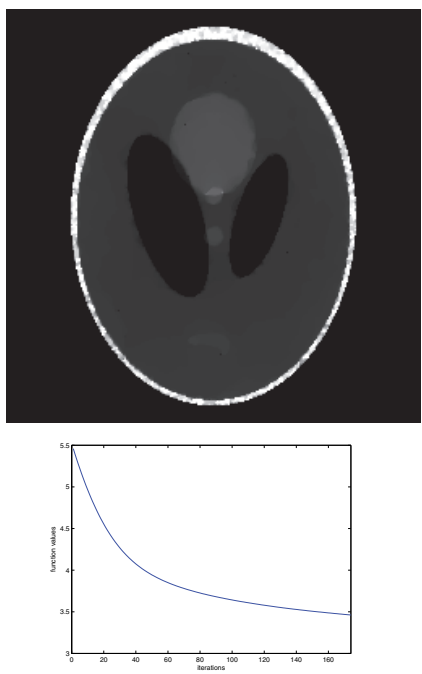

(e)

Figure 5. Results of different methods when removing the multiplicative noise with $K=6$. Bottom row: Plots of the objective function values versus iterations. (a) Noisy Phantom, (b) PPB filter, (c) AA method $(\lambda=0.13)$, (d) RLO method $(\lambda=0.13)$, (e) our method $(\lambda=0.11$ and $\alpha=4)$.

8. Appendix I: Proof of Proposition 3.4. In order to prove Proposition 3.4, we need the following lemma.

Lemma 8.1. Let $B(K)$ denote the Beta-function $B(K, K)$ with $K \geq 1$, i.e., $B(K):=\frac{\Gamma^{2}(K)}{\Gamma(2 K)}$. Then, we have

$$
\frac{B\left(K-\frac{1}{2}\right)}{B(K)}=2+\frac{1}{2 K}+\mathcal{O}\left(\frac{1}{K^{2}}\right)
$$

Proof. Based on a property of the Gamma-function,

$$
\frac{\Gamma(x+1)}{\Gamma(x)}=x \quad \text { for } x>0,
$$




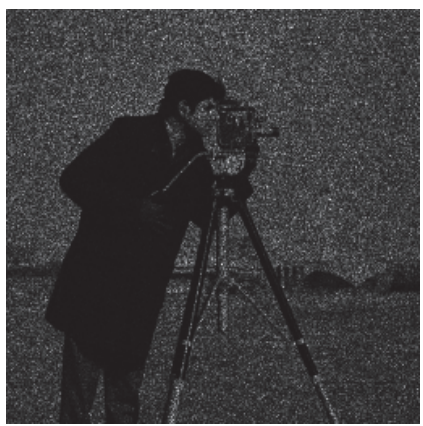

(a)
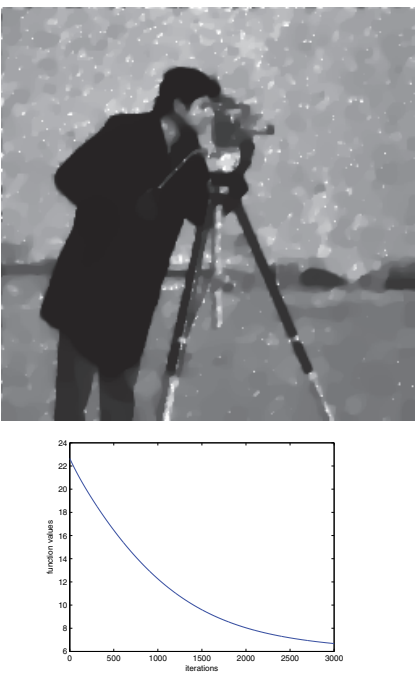

(c)
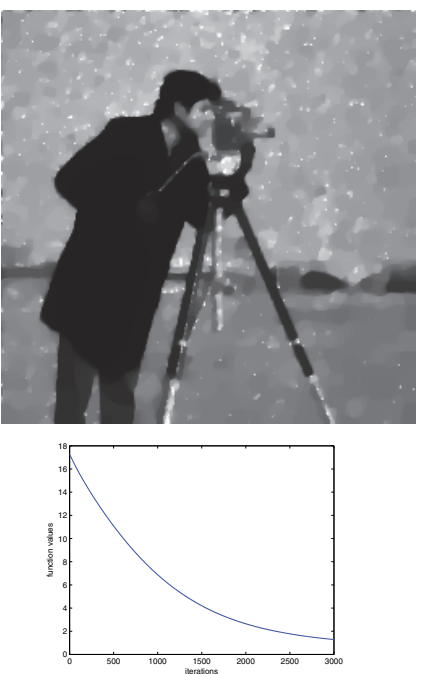

(d)

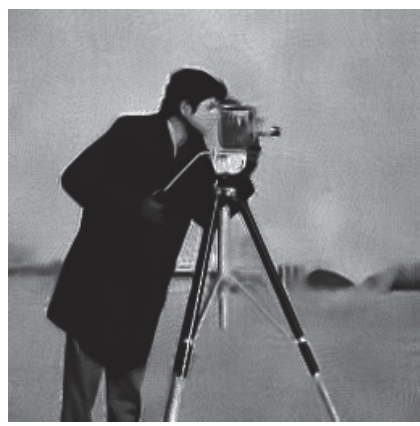

(b)
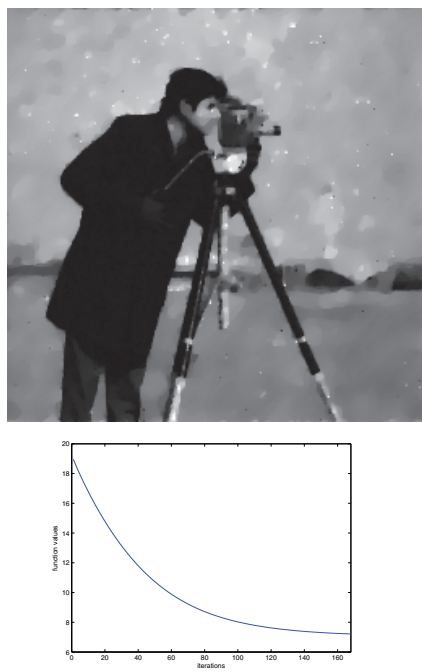

(e)

Figure 6. Results of different methods when removing the multiplicative noise with $K=6$. Bottom row: Plots of the objective function values versus iterations. (a) Noisy Cameraman, (b) PPB filter, (c) AA method $(\lambda=0.2)$, (d) RLO method ( $\lambda=0.2)$, (e) our method $(\lambda=0.16$ and $\alpha=16)$.

we readily obtain

$$
\log \left(\frac{B\left(K-\frac{1}{2}\right)}{2 B(K)}\right)=\log \left(K-\frac{1}{2}\right)+2\left(\log \Gamma\left(K-\frac{1}{2}\right)-\log \Gamma(K)\right) .
$$

Then using the expansion of $\log \Gamma(x)$ with $x>0$ in [2],

$$
\log \Gamma(x)=x \log x-x-\frac{1}{2} \log \frac{x}{2 \pi}+\frac{1}{12 x}+\mathcal{O}\left(\frac{1}{x^{2}}\right),
$$

and the Taylor expansion of $\log (1+x)$ for $x \in(-1,1)$, we get

$$
\begin{aligned}
\log \left(\frac{B\left(K-\frac{1}{2}\right)}{2 B(K)}\right) & =1+(2 K-1) \log \left(1-\frac{1}{2 K}\right)+\frac{1}{12 K\left(K-\frac{1}{2}\right)}+\mathcal{O}\left(\frac{1}{K^{2}}\right) \\
& =\log \left(2+\frac{1}{2 K}\right)+\mathcal{O}\left(\frac{1}{K^{2}}\right) .
\end{aligned}
$$




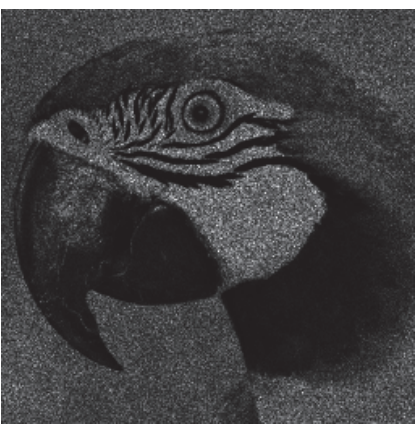

(a)

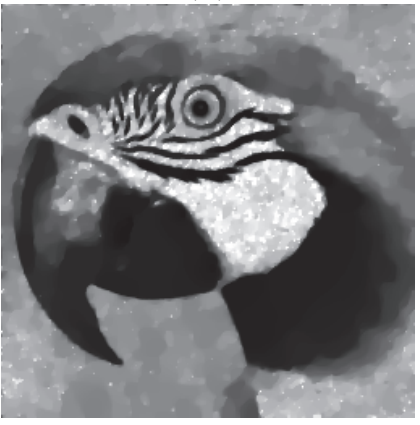

(c)

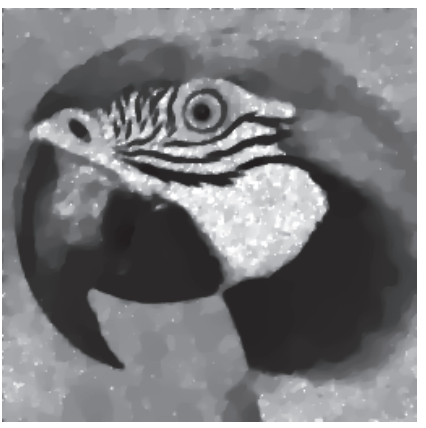

(d)

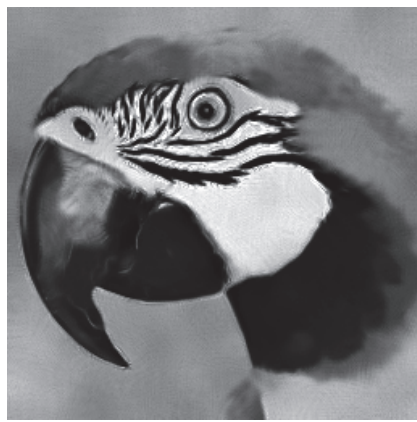

(b)

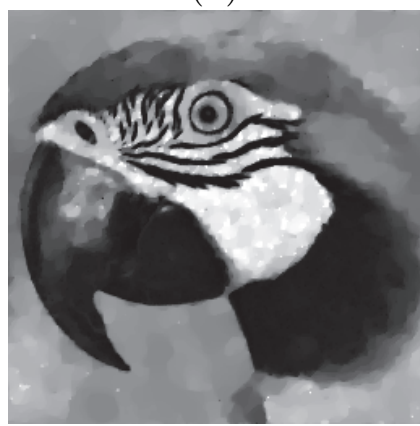

(e)

Figure 7. Images restored by different methods for the image Parrot. (a) Noisy image with $K=10$, (b) PPB filter, (c) AA method $(\lambda=0.14)$, (d) RLO method $(\lambda=0.14)$, (e) our method $(\lambda=0.11$ and $\alpha=16)$.

Table 3

The comparisons of PSNR values, the number of iterations, and CPU-time in seconds for different methods for deblurring with denoising.

\begin{tabular}{|c||c||c|c|c||c|c|c|}
\hline \multirow{2}{*}{\multicolumn{1}{|c||}{ Images }} & \multirow{2}{*}{ Methods } & \multicolumn{3}{c||}{ Motion blur } & \multicolumn{3}{c|}{ Gaussian blur } \\
\cline { 3 - 8 } Phantom & PSNR(dB) & IIter & Time(s) & PSNR(dB) & $\sharp$ Iter & Time(s) \\
\hline \multirow{3}{*}{ Cameraman } & AA & 22.58 & 8000 & 223.36 & 21.12 & $10^{4}$ & 289.23 \\
& RLO & 22.28 & 7000 & 216.14 & 20.81 & $10^{4}$ & 311.00 \\
& Ours & 24.68 & 182 & 84.78 & 22.59 & 331 & 152.85 \\
\hline \multirow{3}{*}{ Parrot } & AA & 22.36 & 8000 & 224.71 & 21.36 & $10^{4}$ & 298.34 \\
& RLO & 22.28 & 8000 & 248.93 & 21.31 & $10^{4}$ & 313.67 \\
& Ours & 22.99 & 200 & 91.93 & 21.85 & 293 & 119.09 \\
\hline & AA & 22.35 & 9000 & 263.82 & 21.35 & $10^{4}$ & 296.58 \\
& RLO & 22.15 & 9000 & 285.12 & 21.23 & $10^{4}$ & 317.76 \\
& Ours & 23.18 & 216 & 100.86 & 22.08 & 223 & 103.92 \\
\hline
\end{tabular}

Further, in view of the Taylor expansion of $e^{x}$, we have

$$
\frac{B\left(K-\frac{1}{2}\right)}{B(K)}=\left(2+\frac{1}{2 K}\right) \exp \left(\mathcal{O}\left(\frac{1}{K^{2}}\right)\right)=2+\frac{1}{2 K}+\mathcal{O}\left(\frac{1}{K^{2}}\right)
$$

Now we are ready to prove Proposition 3.4, as follows.

Copyright (c) by SIAM. Unauthorized reproduction of this article is prohibited. 


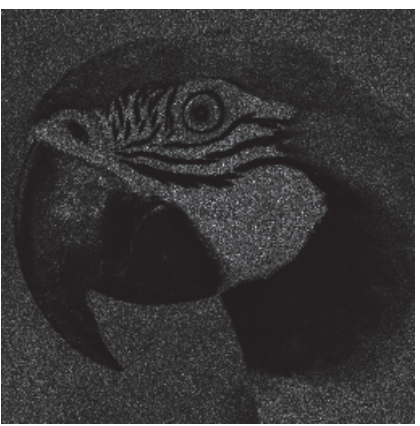

(a)

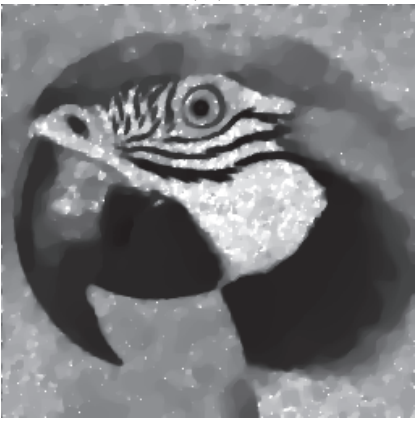

(c)

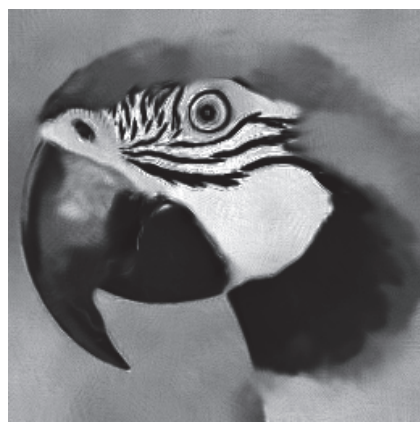

(b)

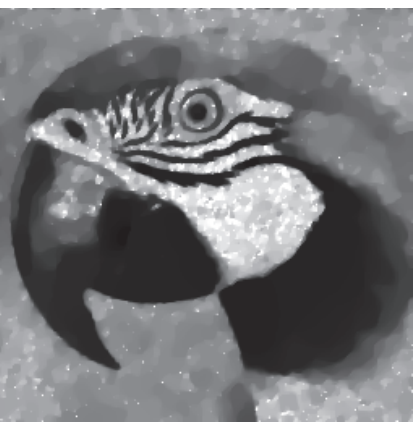

(d)

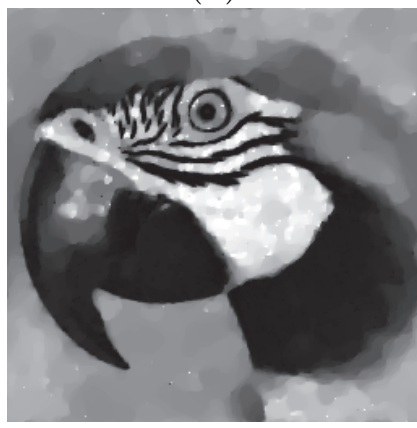

(e)

Figure 8. Images restored by different methods for the image Parrot. (a) Noisy image with $K=6$, (b) PPB filter, (c) AA method ( $\lambda=0.18)$, (d) RLO method ( $\lambda=0.18)$, (e) our method $(\lambda=0.12$ and $\alpha=8)$.

Proof.

(i) Based on (3.2) and $\theta=\frac{1}{K}$, we have

$$
\int_{-\infty}^{+\infty} p_{Y}(y) \log p_{Y}(y) \mathrm{d} y=\int_{0}^{+\infty} p_{Y}(y)\left(\log \frac{2}{\theta^{K} \Gamma(K)}-(2 K+1) \log y-\frac{K}{y^{2}}\right) \mathrm{d} y .
$$

Let $x=\frac{1}{y^{2}}$. Note that the mean of $\eta$ equals 1 ; then we get

$$
\begin{aligned}
\int_{-\infty}^{+\infty} p_{Y}(y) \log p_{Y}(y) \mathrm{d} y & =\int_{-\infty}^{+\infty} p_{\eta}(x)\left(\log \frac{2}{\theta^{K} \Gamma(K)}+\frac{2 K+1}{2} \log x-K x\right) \mathrm{d} x \\
& =\log \frac{2}{\theta^{K} \Gamma(K)}+\frac{2 K+1}{2}(\psi(K)-\log K)-K \\
& =\log 2-\log (\sqrt{K} \Gamma(K))+\frac{2 K+1}{2} \psi(K)-K,
\end{aligned}
$$

where we use a property of the Gamma distribution, $\mathbb{E}_{\eta}(\log x)=\psi(K)-\log K$ (Theorem 2.1 in [33]) and recall that $\psi(K):=\frac{\mathrm{d} \log \Gamma(K)}{\mathrm{d} K}$ is the digamma function (see [2]). 


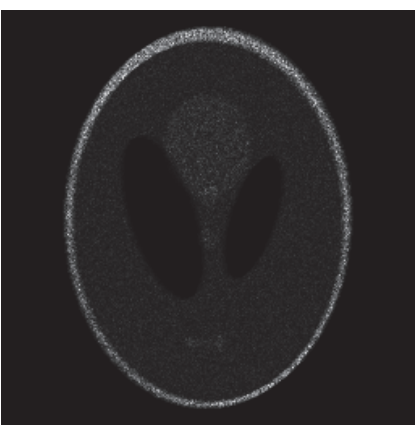

(a)
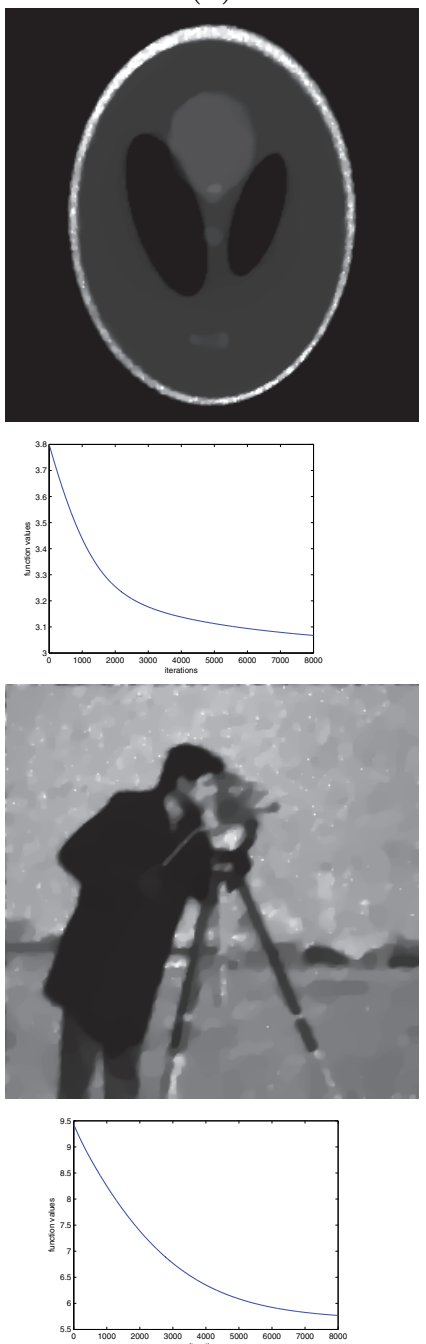

(c)
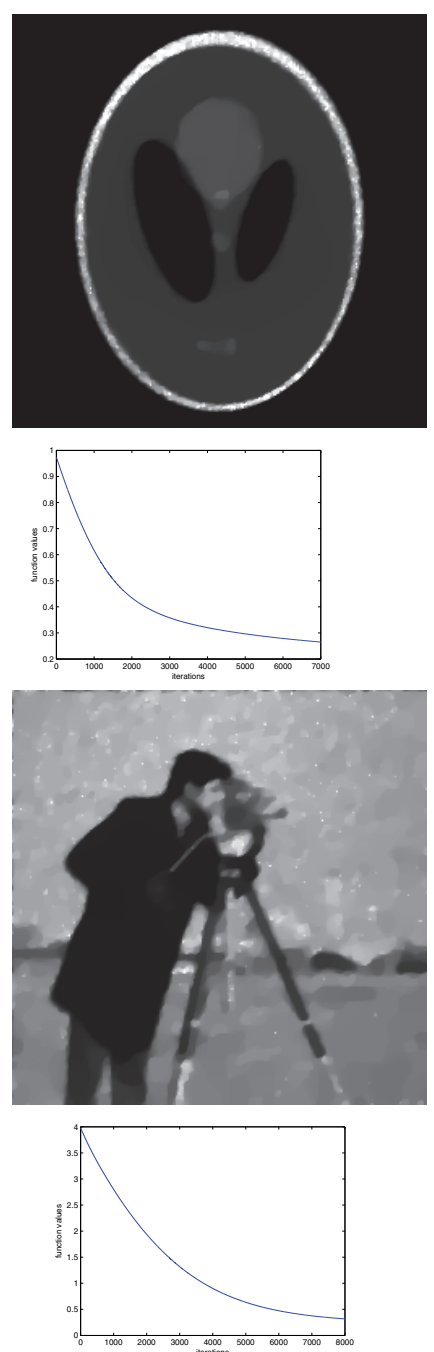

(d)

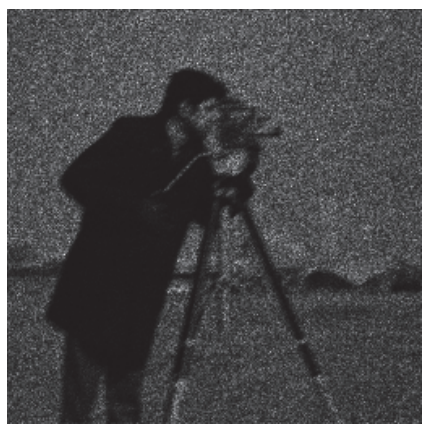

(b)
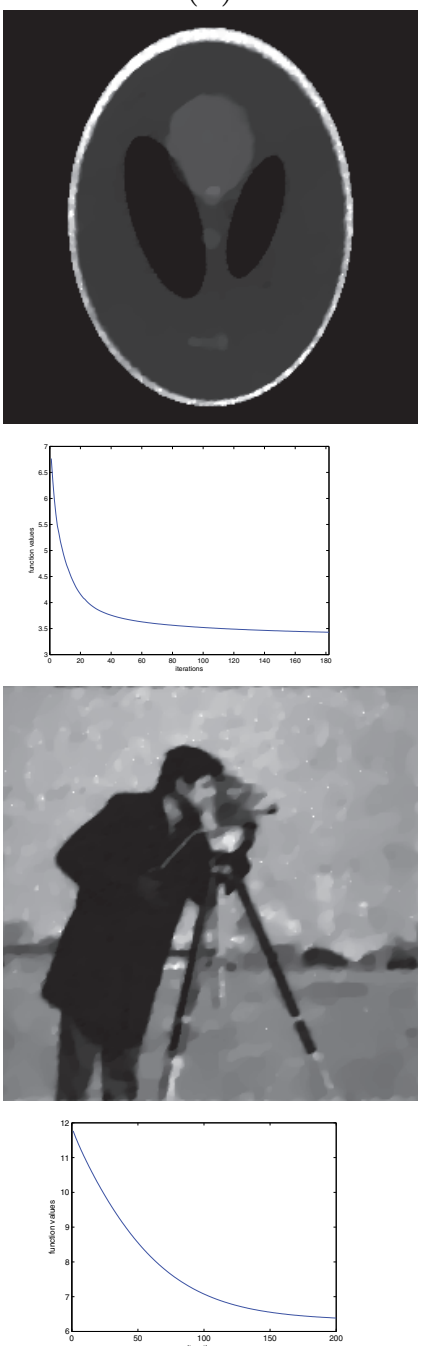

(e)

Figure 9. Results of different methods when restoring the degraded images corrupted by motion blur and then multiplicative noise with $K=10$. Row 1: Degraded images. Rows 2 and 4: Restored images for different methods. Rows 3 and 5: Plots of the objective function values versus iterations. (a) Degraded Phantom, (b) degraded Cameraman, (c) AA method (row 2: $\lambda=0.05$; row 4: $\lambda=0.06$ ), (d) RLO method (row 2: $\lambda=0.05$; row 4: $\lambda=0.06$ ), (e) our method (row $2: \lambda=0.09$ and $\alpha=16$; row $4: \lambda=0.09$ and $\alpha=16$ ). 


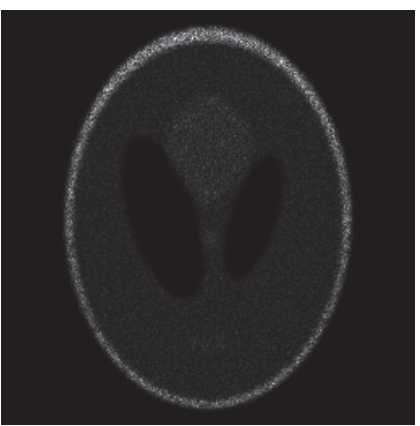

(a)
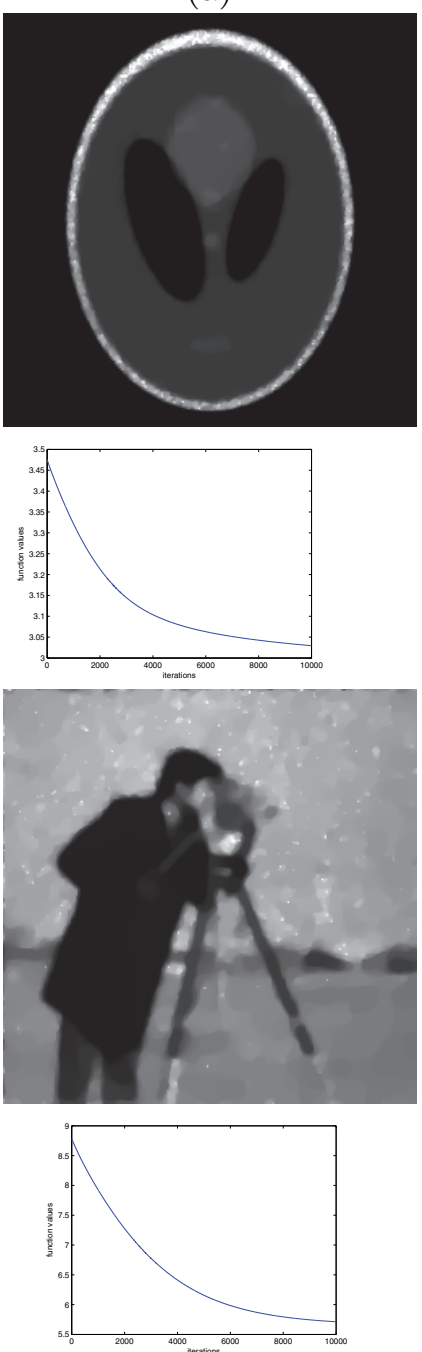

(c)
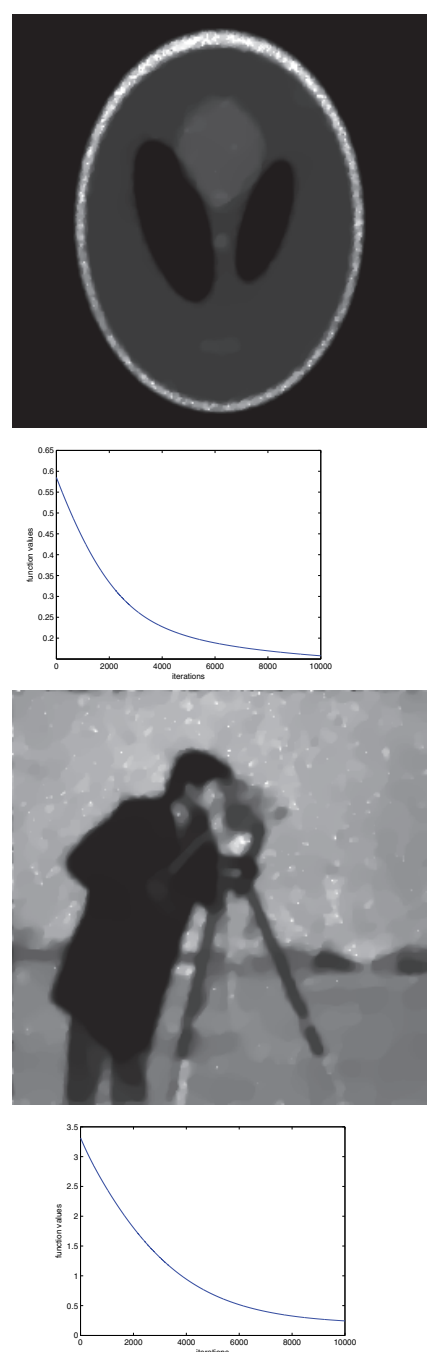

(d)

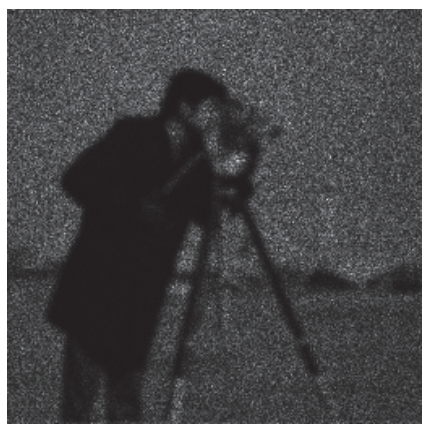

(b)
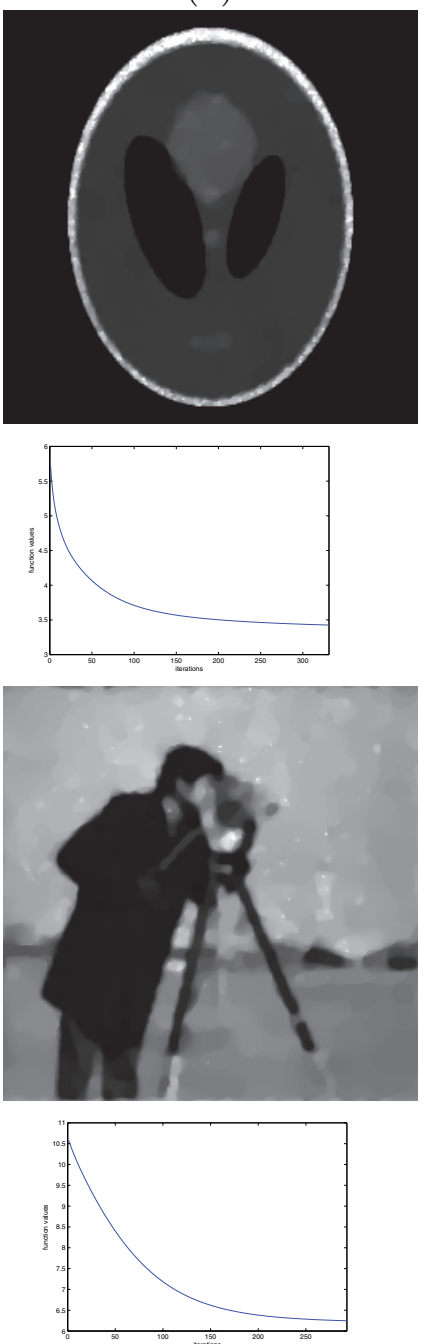

(e)

Figure 10. Results of different methods when restoring the degraded images corrupted by Gaussian blur and then multiplicative noise with $K=10$. Row 1: Degraded images. Rows 2 and 4: Restored images for different methods. Rows 3 and 5: Plots of the objective function values versus iterations. (a) Degraded Phantom, (b) degraded Cameraman, (c) AA method (row 2: $\lambda=0.03$; row 4: $\lambda=0.05$ ), (d) RLO method (row 2: $\lambda=0.03$; row 4: $\lambda=0.05$ ), (e) our method (row $2: \lambda=0.07$ and $\alpha=16$; row $4: \lambda=0.07$ and $\alpha=16$ ).

Copyright $@$ by SIAM. Unauthorized reproduction of this article is prohibited. 


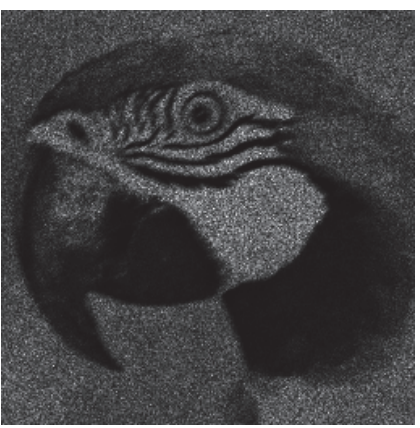

(a)

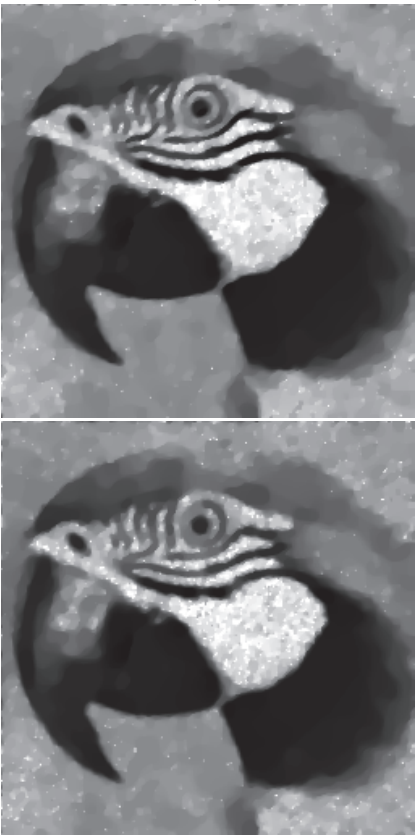

(c)

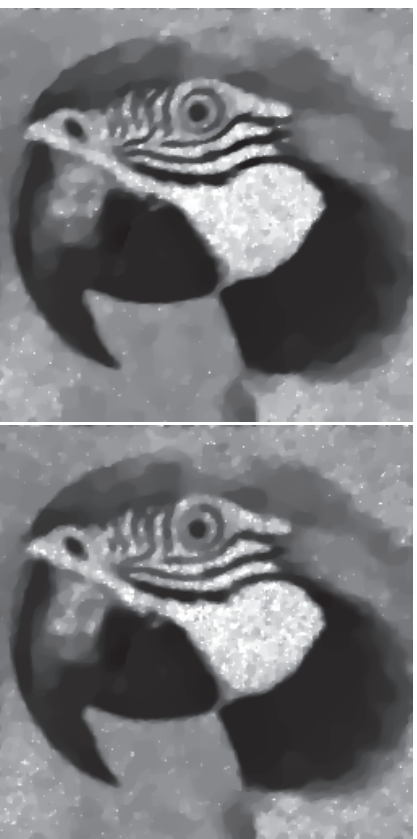

(d)

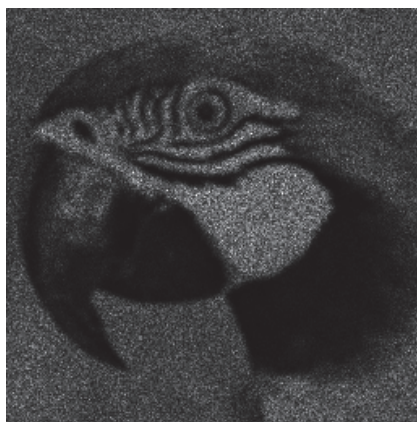

(b)

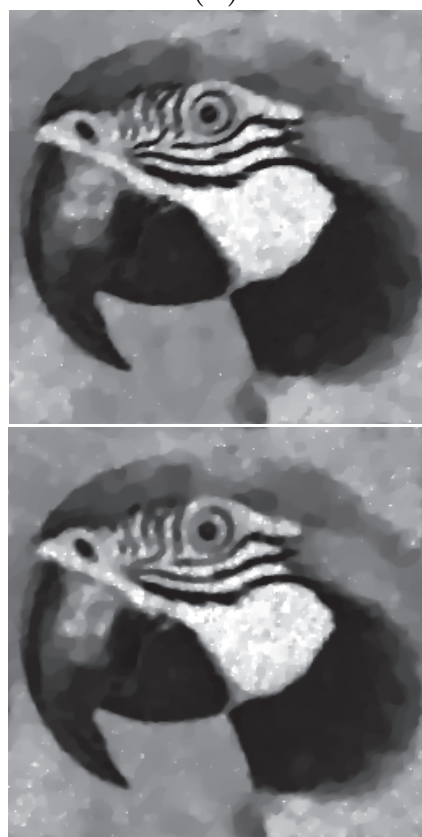

(e)

Figure 11. Results for different methods for the image Parrot blurred by different kernels and then corrupted by multiplicative noise with $K=10$ (row 2: by motion blur; row 3: by Gaussian blur). (a) Image degraded by motion blur, (b) image degraded by Gaussian blur, (c) AA method (row 2: $\lambda=0.05$; row 3: $\lambda=0.04$ ), (d) $R L O$ method (row 2: $\lambda=0.05$; row 3: $\lambda=0.04$ ), (e) our method (row 2: $\lambda=0.08$ and $\alpha=16$; row $3: \lambda=0.07$ and $\alpha=16)$.

(ii) Based on the PDFs of $Y$ and the Gaussian distribution $\mathcal{N}\left(\mu_{K}, \sigma_{K}^{2}\right)$, we have

$$
\begin{aligned}
\int_{-\infty}^{+\infty} & p_{Y}(y) \log p_{\mathcal{N}\left(\mu_{K}, \sigma_{K}^{2}\right)}(y) \mathrm{d} y \\
& =\int_{-\infty}^{+\infty} p_{Y}(y) \log \left(\frac{1}{\sqrt{2 \pi} \sigma_{K}} e^{-\frac{\left(y-\mu_{K}\right)^{2}}{2 \sigma_{K}^{2}}}\right) \mathrm{d} y \\
& =-\int_{-\infty}^{+\infty} p_{Y}(y)\left(\log \left(\sqrt{2 \pi} \sigma_{K}\right)+\frac{\left(y-\mu_{K}\right)^{2}}{2 \sigma_{K}^{2}}\right) \mathrm{d} y \\
& =-\log \left(\sqrt{2 \pi} \sigma_{K}\right) \int_{-\infty}^{+\infty} p_{Y}(y) \mathrm{d} y-\frac{1}{2 \sigma_{K}^{2}} \int_{-\infty}^{+\infty} p_{Y}(y)\left(y-\mu_{K}\right)^{2} \mathrm{~d} y \\
& =-\frac{1}{2} \log \left(2 \pi e \sigma_{K}^{2}\right),
\end{aligned}
$$

Copyright (C) by SIAM. Unauthorized reproduction of this article is prohibited. 
where we have utilized the definition of the variance, i.e.,

$$
\int_{-\infty}^{\infty} p_{Y}(y)\left(y-\mu_{K}\right)^{2} \mathrm{~d} y=\sigma_{K}^{2} .
$$

(iii) In view of the results in (i) and (ii), using the approximation of $\psi(x)$ in [2],

$$
\psi(x)=\log x-\frac{1}{2 x}+\mathcal{O}\left(\frac{1}{x^{2}}\right),
$$

we have

$$
\begin{aligned}
D_{\mathrm{KL}}\left(Y \| \mathcal{N}\left(\mu_{K}, \sigma_{K}^{2}\right)\right)= & \log 2-\log (\sqrt{K} \Gamma(K)) \\
& +\frac{2 K+1}{2} \psi(K)-K+\frac{1}{2} \log \left(2 \pi e \sigma_{K}^{2}\right) \\
= & \log 2-\log (\sqrt{K} \Gamma(K))+\frac{2 K+1}{2}\left(\log K-\frac{1}{2 K}\right) \\
& -K+\frac{1}{2} \log \left(2 \pi e \sigma_{K}^{2}\right)+\mathcal{O}\left(\frac{1}{K}\right) \\
= & \log 2+\frac{1}{2} \log K \sigma_{K}^{2}+\mathcal{O}\left(\frac{1}{K}\right) .
\end{aligned}
$$

Here, we obtain (8.4) by simplifying $D_{\mathrm{KL}}\left(Y \| \mathcal{N}\left(\mu_{K}, \sigma_{K}^{2}\right)\right)$ with (8.3).

Moreover, based on the results in Proposition 3.1, we have

$$
\begin{aligned}
\sigma_{K}^{2} & =\mathbb{E}\left(Y^{2}\right)-\mathbb{E}^{2}(Y) \\
& =\frac{K \Gamma(K-1)}{\Gamma(K)}-\frac{K \Gamma^{2}\left(K-\frac{1}{2}\right)}{\Gamma^{2}(K)} \\
& =\frac{K}{K-1}-\frac{K}{2 K-1} \frac{\Gamma^{2}\left(K-\frac{1}{2}\right)}{\Gamma(2 K-1)} \frac{\Gamma(2 K)}{\Gamma^{2}(K)} \\
& =\frac{K}{2 K-1}-\frac{K}{2 K-1} \frac{B\left(K-\frac{1}{2}\right)}{B(K)} .
\end{aligned}
$$

Substituting the result (8.5) into (8.4) and applying the expansion in Lemma 8.1, we get

$$
\begin{aligned}
& D_{\mathrm{KL}}\left(Y \| \mathcal{N}\left(\mu_{K}, \sigma_{K}^{2}\right)\right) \\
= & \log 2+\frac{1}{2} \log \left(\frac{K^{2}}{K-1}-\frac{K^{2}}{2 K-1}\left(2+\frac{1}{2 K}+\mathcal{O}\left(\frac{1}{K^{2}}\right)\right)\right)+\mathcal{O}\left(\frac{1}{K}\right) \\
= & \log 2+\frac{1}{2} \log \left(\frac{1}{4}+\mathcal{O}\left(\frac{1}{K}\right)\right)+\mathcal{O}\left(\frac{1}{K}\right) \\
= & \mathcal{O}\left(\frac{1}{K}\right)
\end{aligned}
$$

which yield the assertion. 
Acknowledgment. The authors would like to thank the reviewers for the careful reading of the manuscript and the insightful and constructive comments.

\section{REFERENCES}

[1] C. Deledalle, Probabilistic patch-based filter (PPB), image analysis software, http://www.math.ubordeaux1.fr/ cdeledal/ppb.php.

[2] M. Abramowitz and I. Stegun, Handbook of Mathematical Functions with Formulas, Graphs, and Mathematical Tables, 7th ed., Appl. Math. Ser. 55, National Bureau of Standards, Gaithersburg, MD, 1968.

[3] L. Ambrosio, N. Fusco, And D. Pallara, Functions of Bounded Variation and Free Discontinuity Problem, Oxford University Press, London, 2000.

[4] G. Andrews, R. Askey, And R. Roy, Special Functions, Cambridge University Press, Cambridge, UK, 2001.

[5] G. Aubert And J.-F. Aujol, A variational approach to removing multiplicative noise, SIAM J. Appl. Math., 68 (2008), pp. 925-946.

[6] G. Aubert And P. Kornprobst, Mathematical Problems in Image Processing. Partial Differential Equations and the Calculus of Variations, Appl. Math. Sci. 147, Springer, New York, 2006.

[7] P. Bickel and K. Doksum, Mathematical Statistics: Basic Ideas and Selected Topics, Vol. 1, 2nd ed., Pearson, NJ, 2007.

[8] J. Biouchs-Dias AND M. Figueiredo, Total variation restoration of speckled images using a splitBregman algorithm, in Proceedings of the IEEE International Conference on Image Processing, 2009, pp. 3717-3720.

[9] J. Biouchs-Dias And M. Figueiredo, Multiplicative noise removal using variable splitting and constrained optimization, IEEE Trans. Image Process., 19 (2010), pp. 1720-1730.

[10] A. Bovik, Handbook of Image and Video Processing, Academic Press, New York, 2000.

[11] S. Boyd, N. Parikh, E. Chu, B. Peleato, and J. Eckstein, Distributed optimization and statistical learning via the alternating direction method of multipliers, Found. and Trends Mach. Learning, 3 (2010), pp. 1-122.

[12] J. CAI, R. Chan, AND Z. Shen, A framelet-based image inpainting algorithm, Appl. Comput. Harmon. Anal., 24 (2008), pp. 131-149.

[13] A. Chambolle, An algorithm for total variation minimization and application, J. Math. Imaging Vis., 20 (2004), pp. 89-97.

[14] A. Chambolle and P. Lions, Image recovery via total variation minimization and related problems, Numer. Math., 76 (1997), pp. 167-188.

[15] A. Chambolle And T. Pock, A first-order primal-dual algorithm for convex problems with applications to imaging, J. Math. Imaging Vis., 40 (2011), pp. 120-145.

[16] T. F. Chan And J. Shen, Image Processing and Analysis: Variational, PDE, Wavelet, and Stochastic Methods, SIAM, Philadelphia, 2005.

[17] T. Chartrand And T. Asaki, A variational approach to reconstructing images corrupted by Poisson noise, J. Math. Imaging Vis., 27 (2007), pp. 257-263.

[18] C. Chaux, J.-C. Pesquet, and N. Pustelnik, Nested iterative algorithms for convex constrained image recovery problems, SIAM J. Imaging Sci., 2 (2009), pp. 730-762.

[19] P. Combettes And J. Pesquet, A Douglas-Rachford splitting approach to nonsmooth convex variational signal recovery, IEEE J. Selected Topics Signal Process., 1 (2007), pp. 564-574.

[20] C.-A. Deledalle, L. Denis, And F. Tupin, Iterative weighted maximum likelihood denoising with probabilistic patch-based weights, IEEE Trans. Image Process., 18 (2009), pp. 2661-2672.

[21] Y. Dong, M. Hintermüller, And M. Neri, An efficient primal-dual method for $L_{1} T V$ image restoration, SIAM J. Imaging Sci., 2 (2009), pp. 1168-1189.

[22] S. Durand, J. FAdili, AND M. NikolOva, Multiplicative noise removal using L1 fidelity on frame coefficients, J. Math. Imaging Vis., 36 (2010), pp. 201-226.

[23] J. Einmahl, Poisson and Gaussian approximation of weighted local empirical processes, Stochastic Process Appl., 70 (1997), pp. 31-58.

Copyright $\odot$ by SIAM. Unauthorized reproduction of this article is prohibited. 
[24] E. Esser, X. Zhang, AND T. F. ChAn, A general framework for a class of first order primal-dual algorithms for convex optimization in imaging science, SIAM J. Imaging Sci., 3 (2010), pp. 10151046.

[25] M. Figueiredo And J. Bioucas-Dias, Restoration of Poissonian images using alternating direction optimization, IEEE Trans. Image Process., 19 (2010), pp. 3133-3145.

[26] G. Gilbon And S. Osher, Nonlocal operators with applications to image processing, Multiscale Model. Simul., 7 (2008), pp. 1005-1028.

[27] E. Giusti, Minimal Surfaces and Functions of Bounded Variation, Birkhäuser Boston, Cambridge, MA, 1984.

[28] T. Goldstein And S. Osher, The split Bregman algorithm for L1-regularized problems, SIAM J. Imaging Sci., 2 (2009), pp. 323-343.

[29] G. Grimmett and D. Welsh, Probability: An Introduction, Oxford Science Publications, London, 1986.

[30] J. Hadamard, Sur les problèmes aux dérivées partielles et leur signification physique, Princeton Univ. Bull., 13 (1902), pp. 49-52.

[31] Y. Huang, L. Moisan, M. NG, And T. Zeng, Multiplicative noise removal via a learned dictionary, IEEE Trans. Image Process., 21 (2012), pp. 4534-4543.

[32] Y.-M. Huang, M. K. NG, And Y.-W. Wen, A new total variation method for multiplicative noise removal, SIAM J. Imaging Sci., 2 (2009), pp. 20-40.

[33] M. Khodabin and A. Ahmadabadi, Some properties of generalized gamma distribution, Math. Sci., 4 (2010), pp. 9-28.

[34] P. Kornprobst, R. Deriche, AND G. Aubert, Image sequence analysis via partial differential equations, J. Math. Imaging Vis., 11 (1999), pp. 5-26.

[35] T. Le, R. Chartrand, AND T. AsAKI, A variational approach to reconstructing images corrupted by Poisson noise, J. Math. Imaging Vis., 27 (2007), pp. 257-263.

[36] F. Li, M. K. NG, AND C. Shen, Multiplicative noise removal with spatially varying regularization parameters, SIAM J. Imaging Sci., 3 (2010), pp. 1-20.

[37] T. Pock, D. Cremers, H. Bischof, and A. Chambolle, An algorithm for minimizing the MumfordShah functional, in Proceedings of the 12th IEEE International Conference on Computer Vision, 2009, pp. 1133-1140.

[38] B. Quinn AND H. MacGillivray, Normal approximations to discrete unimodal distributions, J. Appl. Probab., 23 (1986), pp. 1013-1018.

[39] L. Rudin, P. LiOns, AND S. Osher, Multiplicative denoising and deblurring: Theory and algorithms, in Geometric Level Set Methods in Imaging, Vision, and Graphics, S. Osher and N. Paragios, Springer, New York, 2003, pp. 103-119.

[40] L. Rudin, S. OsheR, AND E. FAtemi, Nonlinear total variation based noise removal algorithms, Phys. D, 60 (1992), pp. 259-268.

[41] J. Shi And S. Osher, A nonlinear inverse scale space method for a convex multiplicative noise model, SIAM J. Imaging Sci., 1 (2008), pp. 294-321.

[42] G. Steidl and T. Teuber, Removing multiplicative noise by Douglas-Rachford splitting methods, J. Math. Imaging Vis., 36 (2010), pp. 168-184.

[43] T. Teuber And A. LANG, Nonlocal filters for removing multiplicative noise, in Scale Space and Variational Methods in Computer Vision 6667, Springer, New York, 2011, pp. 50-61.

[44] Y. Xiao, T. Zeng, J. Yu, And M. NG, Restoration of images corrupted by mixed Gaussian-impulse noise via $l_{1}-l_{0}$ minimization, Pattern Recognition, 44 (2011), pp. 1708-1728.

[45] T. Zeng and M. NG, On the total variation dictionary model, IEEE Trans. Image Process., 19 (2010), pp. 821-825.

Copyright (C) by SIAM. Unauthorized reproduction of this article is prohibited. 